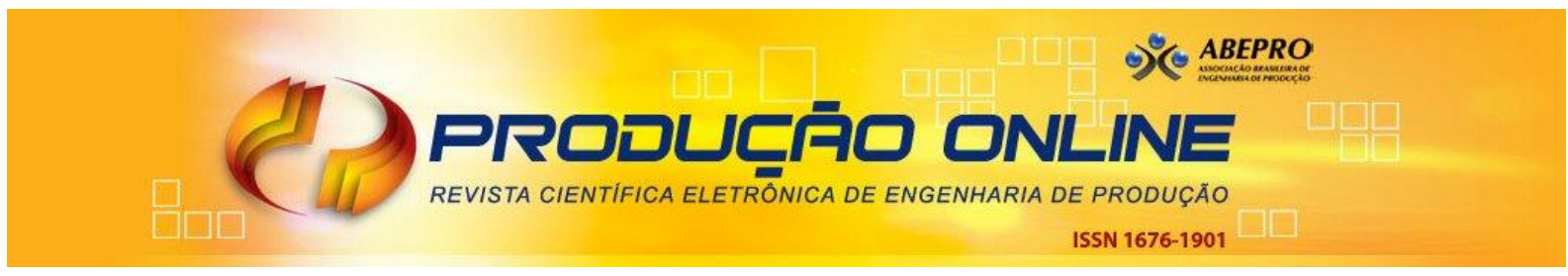

\title{
CAPACIDADES COLABORATIVAS E CRIAÇÃO DE VALOR: REVISÃO SISTE- MÁTICA E AGENDA DE PESQUISA
}

\section{COLLABORATIVE CAPABILITIES AND VALUE CREATION: SYSTEMATIC REVIEW AND RESEARCH AGENDA}

\author{
Bruna Medeiros da Nóbrega* E-mail: brunamnobrega@hotmail.com \\ Nathalia Rodarte de Moura*E-mail: nathaliarodartemoura@gmail.com \\ Cláudia Fabiana Gohr* E-mail: claudiagohr@ct.ufpb.br \\ Juliana Maria Silva Gonçalves* E-mail: julianamgoncalvess@gmail.com \\ *Programa de Pós-Graduação em Engenharia de Produção, Departamento de Engenharia de \\ Produção, Universidade Federal da Paraíba (UFPB), João Pessoa, PB.
}

Resumo: Para que empresas colaborem entre si e criem valor conjunto, é necessário o desenvolvimento de capacidades. No entanto, há uma lacuna sobre quais capacidades colaborativas devem ser desenvolvidas para criar valor conjunto. Este artigo tem como principal objetivo identificar atributos de capacidade colaborativa e criação de valor que influenciam no desenvolvimento desses aspectos, utilizando-se como método a Revisão Sistemática da Literatura. A literatura foi analisada por meio de análise de conteúdo. Foi possível identificar 15 atributos de capacidade colaborativa e 14 atributos de criação de valor. Verificou-se que os atributos de capacidade colaborativa podem influenciar no processo de criação de valor conjunto, como por exemplo, alinhamento estratégico, aprendizado, atributos da rede e organizacionais, compartilhamento de conhecimento e de recursos, comprometimento, comunicação, confiança, desempenho, governança relacional, novas parcerias e relacionamento longo. Dentre as contribuições do artigo, destacam-se o avanço do conhecimento sobre a temática ainda não explorada na literatura, os atributos de capacidade colaborativa que precisam ser desenvolvidos em um relacionamento interorganizacional para possibilitar a criação de valor, as oportunidades de pesquisas identificadas na literatura e as que podem surgir em função desta pesquisa.

Palavras-Chave: Capacidade colaborativa. Criação de valor. Revisão sistemática da literatura.

Abstract: The development of capabilities is needed so that enterprises collaborate and create joint value. However, there is still a lack about which collaborative capabilities should be developed to create joint value. Through a Systematic Literature Review, this paper aims to identify collaborative capability and joint value creation attributes that influence in these aspects. The literature was analyzed towards a content analysis. It was possible to identify 15 collaborative capability attributes and 14 value creation attributes. It was verified that the collaborative capability attributes influence on the joint value creation process. Among the contributions of this paper, the advance of the knowledge about not explored themes in the literature, the collaborative capability attributes that must be developed by interorganizational relationships to make possible the creation of joint value, the opportunities for research identified in the literature and the ones that can emerge in function of this paper are highlighted.

Keywords: Collaborative capability. Value creation. Systematic Literature Review. 


\section{INTRODUÇÃO}

Relacionamentos interorganizacionais podem ocorrer no contexto de clusters (MOLINA-MORALES; MARTÍNEZ-FERNÁNDEZ, 2009), cadeias de suprimentos (STADTLER, 2009; SUBRAMANI, 2004), alianças estratégicas (PARUNG; BITITCI, 2006, 2008; SARKAR et al., 2001), redes (MATINHEIKKI et al., 2016; SAUNILA et al., 2017), aglomerações (TELLER; ALEXANDER; FLOH, 2016), configuração multistakeholder (RÜHLI et al., 2017), etc. Nesses relacionamentos, a colaboração é uma importante estratégia e significa um relacionamento próximo entre os parceiros (PARUNG; BITITCI, 2006, 2008). Em relacionamentos interorganizacionais, empresas compartilham informações, conhecimento, riscos e benefícios (PARUNG; BITITCI, 2006, 2008). Assim, a colaboração entre empresas resulta, por exemplo, na redução de custos, no aumento da receita, na sinergia de recursos, no aprendizado e na inovação (HANSEN; NOHRIA, 2004; JAP, 1999; LADO; BOYD; HANLON, 1997; SIMATUPANG; SRIDHARAN, 2005; TANRIVERDI, 2006).

Para que haja colaboração entre empresas, a confiança é um atributo fundamental, que, aliada ao comprometimento é capaz de assegurar o desenvolvimento de capacidades colaborativas (CC) (CHEUNG; MYERS; MENTZER, 2010).

Capacidades colaborativas podem ser caracterizadas como um tipo de capacidade dinâmica, sendo esta originalmente definida como "a capacidade da empresa de integrar, construir e reconfigurar competências internas e externas para abordar ambientes que mudam rapidamente" (TEECE; PISANO; SHUEN, 1997, p. 516 tradução nossa). Assim, a CC é um tipo de capacidade dinâmica capaz de criar, ampliar ou modificar sua base de recursos propositalmente para incluir os recursos de seus parceiros (HELFAT et al., 2007).

Já a literatura de criação de valor (CV) argumenta que valor é algo que adiciona, traz benefícios e vantagens (KAUFFMAN; LI; VAN HECK, 2010). Dessa forma, no contexto da colaboração, a CV está relacionada aos benefícios adquiridos por meio da parceria (CHEUNG; MYERS; MENTZER, 2010; WAGNER; EGGERT; LINDEMANN, 2010)

Em função de sua CC, parceiros trazem recursos únicos e valiosos de modo a potencializarem a CV (SARKAR et al., 2001). Entretanto, as empresas devem estar conscientes dos potenciais riscos da colaboração (GULATI; LAVIE; SINGH, 2009). 
Nesse contexto, o processo de CV tem sido um tema de interesse quando se trata de relacionamentos interorganizacionais. Esse processo tem sido apontado como a razão pela qual as firmas colaboram (DYER; SINGH, 1998). No entanto, quando se alia isso à CC, verifica-se que ainda há lacunas a serem exploradas elucidando algumas questões, tais, como: quais atributos influenciam no desenvolvimento de CC? Como essa capacidade pode influenciar no desenvolvimento de CV? Procurando responder essas perguntas, este artigo tem como objetivo identificar atributos de CC e de CV em relacionamentos interorganizacionais, por meio de uma revisão sistemática da literatura (RSL). A partir da análise dos artigos também foi possível identificar tendências, lacunas e oportunidades para novas pesquisas.

O artigo encontra-se estruturado em cinco seções, incluindo esta introdução. Na próxima, são apresentados os procedimentos metodológicos. A terceira mostra os resultados. Posteriormente a discussão, as lacunas e as sugestões para futuros trabalhos. Em sequência, as conclusões, limitações e sugestões que surgiram a partir desse artigo são apresentadas.

\section{PROCEDIMENTOS METODOLÓGICOS}

Uma RSL compreende desde a definição de um objeto de estudo até o conhecimento sobre o que tem sido discutido nas academias, além de permitir identificar sugestões para trabalhos futuros a partir das lacunas dos trabalhos analisados (ARMITAGE; KEEBLE-ALLEN, 2008). Lacerda; Ensslin e Ensslin (2012) separam a RSL em três etapas: (I) Investigação preliminar, (II) Seleção dos artigos e (III) Análise dos artigos. Essas etapas foram adotadas para o desenvolvimento deste trabalho.

$\mathrm{Na}$ etapa I, a base de dados selecionada foi Web of Science, pois representa relevante contribuição científica para o tema pesquisado. Na sequência, foram escoIhidas algumas palavras-chave e realizado um teste de aderência entre elas, selecionando para o estudo as combinações mais adequadas (Quadro 1). A busca pelas combinações ocorreu no título, nas palavras-chave e no resumo, simultaneamente.

A pesquisa foi realizada em outubro de 2017, na qual um total de 1387 artigos foi encontrado, excluindo-se os artigos de eventos, congressos, livros, capítulos de livros e patentes, a fim de se analisar apenas artigos de revistas científicas. Além 
disso, foram selecionados artigos das seguintes áreas: bussiness, economics, engineering industrial, engineering manufacturing, engineering multidisciplinar, management, operations research management, social sciences, interdisciplinar.

Quadro 1 - Palavras-chave

\begin{tabular}{|c|c|c|}
\hline Parcerias Interorganizacionais & Capacidades Colaborativas & Criação de valor \\
\hline 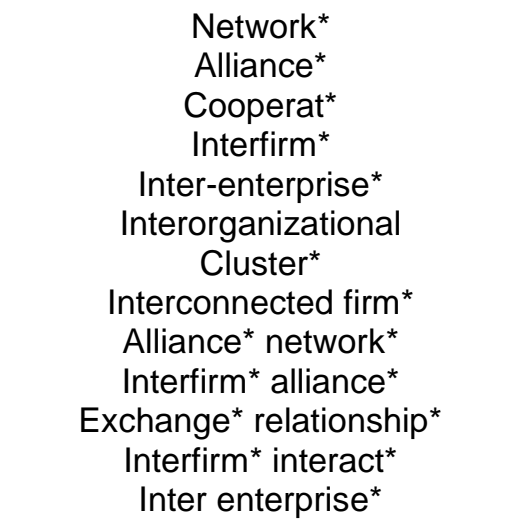 & $\begin{array}{l}\text { Collaborative capacitt } \\
\text { Collaborative capabilit* }^{\star} \\
\text { Collaborative abilit* } \\
\text { Collaborative competenc }{ }^{\star}\end{array}$ & $\begin{array}{c}\text { Value creat* } \\
\text { Join benefit* } \\
\text { Relational advantag* } \\
\text { Strategic assets } \\
\text { Relational rent* } \\
\text { Colaborative advantag* }\end{array}$ \\
\hline
\end{tabular}

* foi utilizado para encontrar palavras no singular e no plural, bem como as variações de escrita.

Na etapa II, seguiram-se nove passos: (1) realizar a busca na base de dados; (2) importar os artigos para o aplicativo EndNote; (3) excluir da amostra as referências duplicatas; (4) ler títulos dos artigos para observar o alinhamento; (5) analisar os artigos pelo seu reconhecimento científico (número de citações dos artigos), sendo estabelecido um valor de corte (esse valor representa a seleção das referências mais citadas até que suas citações representem um valor superior a $85 \%$ de todas as citações obtidas pelos artigos analisados); (6) analisar os artigos mais citados quanto ao alinhamento do seu resumo; (7) analisar os artigos menos citados através de duas condições: (a) os artigos publicados há menos de 2 anos; (b) os artigos publicados há mais de 2 anos deveriam ser de autoria de algum pesquisador já presente no grupo dos artigos alinhados; (8) ler resumos dos artigos que atendesse a uma das condições; (9) ler todos os artigos selecionados e excluir os que não se enquadram na temática. Esses passos estão descritos na Figura 1.

Na etapa III, a amostra de artigos foi analisada utilizando-se o método de análise de conteúdo sendo realizadas uma análise quantitativa e qualitativa. Essa técnica é considerada como uma abordagem sistemática e rigorosa para analisar documentos obtidos ou gerados no decorrer da pesquisa (WHITE; MARSH, 2006). 
Figura 1 - Etapas da Revisão Sistemática

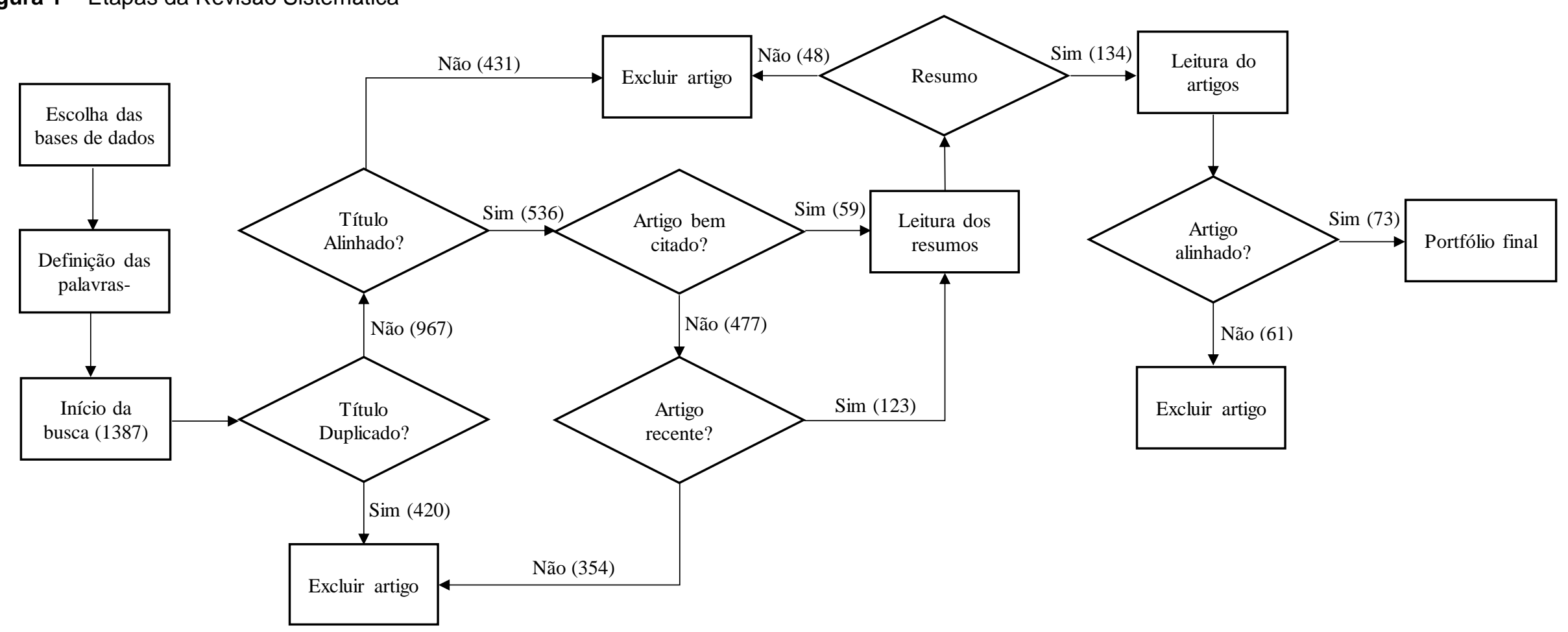




\section{a) Análise quantitativa}

Esse procedimento foi realizado com o portfólio final (Fig.1) através da análise das palavras-chave, da evolução das publicações, dos autores e obras das referências, bem como dos termos, dos métodos de pesquisa e de sua evolução ao longo do tempo, dos relacionamentos interorganizacionais e das teorias mais adotadas. Essa análise foi feita por meio da leitura dos artigos e teve o auxílio do software Bibexcel (ferramenta para extração de dados e combinação de resultados). O diferencial desse é oferecer possibilidade de correlação de termos (extraídos do título ou resumo) e de citações (PERUCCl; CAMPOS, 2016).

\section{b) Análise qualitativa}

A partir da leitura dos 73 artigos, foram identificados os principais atributos capazes de influenciar no desenvolvimento de $\mathrm{CC}$ e de $\mathrm{CV}$ em parcerias interorganizacionais. Também foi possível fazer uma análise aprofundada do tema, identificando tendências, lacunas e oportunidades para novas pesquisas.

\section{RESULTADOS}

\subsection{Visão geral das pesquisas}

Em relação aos anos de publicação (Fig. 2a), em 2016 e 2017 houve um pico na quantidade de trabalhos publicados indicando que se trata de uma temática recente, com forte ascensão em função da alternativa estratégica de adotar relacionamentos colaborativos.

Em relação aos termos utilizados nas palavras-chave (Fig. 2b), 253 expressões foram extraídas, das quais "criação de valor", "rede", "inovação", "relação interorganizacional", "gerenciamento da cadeia de suprimento" e "confiança" apareceram no topo do ranking. Isso mostra que embora $\mathrm{CV}$ tenha sido um tema de interesse quando se trata relacionamentos interorganizacionais (em redes e cadeias de suprimentos), quando aliada à $\mathrm{CC}$, ainda há lacunas a serem exploradas.

Do levantamento realizado sobre os principais autores das referências (Fig. 2c), 474 foram identificados, sendo Dyer, Gulati, Williamson, Kogut, Anderson, Uzzi, Burt, Teece e Powell os mais frequentes. Convém ressaltar que os quatro primeiros são 
autores pioneiros em pesquisas e teorias relacionadas aos relacionamentos interorganizacionais, como a visão relacional (VR) (Dyer), experiências e ganhos provenientes de alianças (Gulati), teoria dos custos de transação (TCT) (Willianson) e networks (Kogut).

Figura 2b - Palavras-chave de maior ocorrência

Figura 2a - Número de publicações por ano
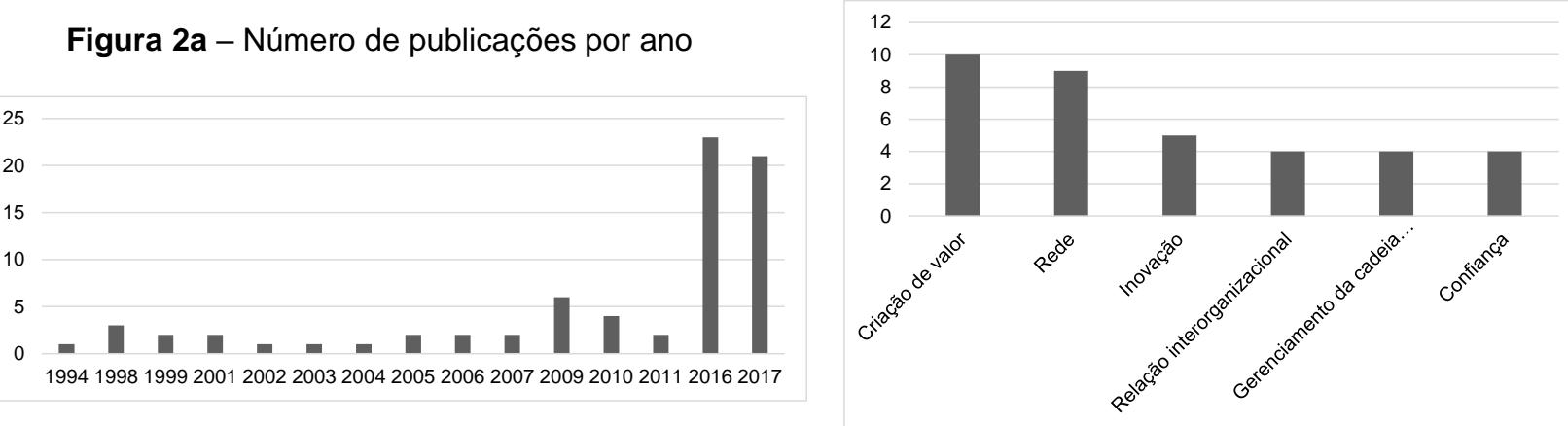

Figura 2c - Principais autores das referências

Figura 2d - Principais obras das referências
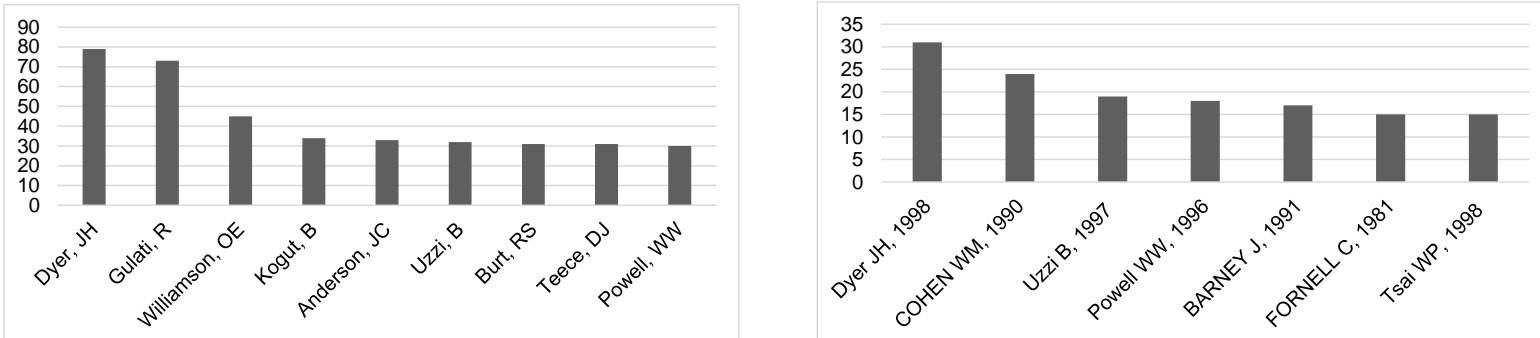

Fonte: Bibexcel (2018)

Com relação às principais obras das referências (Fig. 2d), identificaram-se 496, sendo Dyer J.H. (1998); Cohen W.M. (1990); Uzzi B. (1997); Powell W.W. (1996); Barney J. (1991); Fornell C. (1981); Tsai W.P. (1998) as mais assíduas. Convém ressaltar Dyer como autor e obra mais citada.

Os principais métodos de pesquisa e tipos de arranjos estudados podem ser observados na Tab. 1. Na mesma Tabela, observa-se que dos temas abordados, 59\% dos trabalhos associaram CC à CV, enquanto 37\% utilizaram apenas CC e 4\% CV. O arranjo "não especificado" (Tab. 1) corresponde aos trabalhos que não descreveram o arranjo estudado, utilizando classificações como colaboração, colaboração em projeto, parceria ou relação interorganizacional entre firmas. Além disso, alinhado à literatura, foi possível verificar a ocorrência de relacionamentos interorganizacionais em diferentes contextos.

Os métodos de pesquisa também podem ser visualizados na Fig. 3a, e observa-se que o survey é o mais adotado, com o maior número de trabalhos em 2016 
e 2017, tanto nas pesquisas sobre CC como naquelas que estudam CC e CV de forma intercambiada. O método menos empregado é a modelagem matemática, com apenas um trabalho publicado em 2016. As pesquisas conceituais também são escassas.

Tabela 1 - Características dos artigos

\begin{tabular}{|c|c|c|c|c|c|}
\hline Classificação & Sub-classificação & $\begin{array}{l}\text { Capacidade } \\
\text { Colaborativa }\end{array}$ & $\begin{array}{c}\text { Criação de } \\
\text { Valor }\end{array}$ & $\begin{array}{c}\text { Capacidade } \\
\text { Colaborativa e } \\
\text { Criação de } \\
\text { Valor }\end{array}$ & Total \\
\hline \multirow{4}{*}{$\begin{array}{l}\text { Método de } \\
\text { Pesquisa }\end{array}$} & Conceitual & 3 & 0 & 8 & 11 \\
\hline & Estudo de caso & 5 & 2 & 7 & 14 \\
\hline & Misto & 4 & 1 & 5 & 10 \\
\hline & Modelagem Matemática & 1 & 0 & 0 & 1 \\
\hline \multirow{9}{*}{$\begin{array}{l}\text { Tipo de ar- } \\
\text { ranjo estu- } \\
\text { dado }\end{array}$} & Survey & 14 & 0 & 23 & 37 \\
\hline & Aglomeração & 0 & 0 & 1 & 1 \\
\hline & Aliança* & 6 & 0 & 8 & 14 \\
\hline & Cadeia de Suprimento & 3 & 1 & 7 & 11 \\
\hline & Cluster & 0 & 0 & 1 & 1 \\
\hline & $\begin{array}{c}\text { Configuração multi- } \\
\text { stakeholder }\end{array}$ & 1 & 0 & 0 & 1 \\
\hline & Portfólio de alianças & 1 & 0 & 1 & 2 \\
\hline & Rede* & 3 & 1 & 15 & 19 \\
\hline & Não especificado & 13 & 1 & 10 & 24 \\
\hline \multicolumn{2}{|c|}{ Total de artigos } & 27 & 3 & 43 & 73 \\
\hline
\end{tabular}

* "de/entre empresas", "interorganizacional", "entre firmas", "inter-firma", "público-privada", "de/entre fornecedores" e "de/entre fornecedores e compradores", "de negócios".

Figura 3a - Métodos de pesquisa ao longo do tempo

Figura $\mathbf{3 b}$ - Principais teorias utilizadas
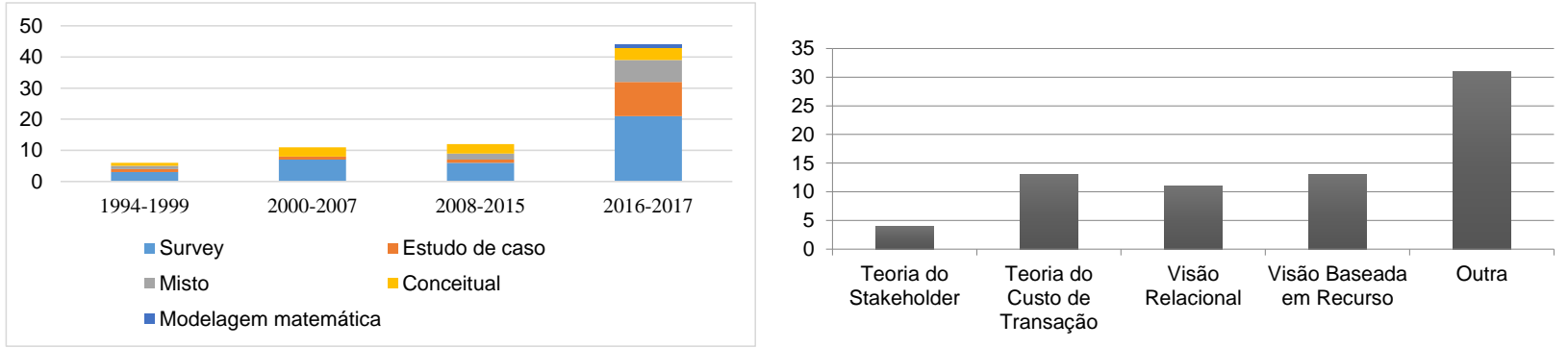

As teorias adotadas são observadas na Fig. 3b, merecendo destaque a Visão Baseada em Recursos e a TCT. Os cálculos para essa análise basearam-se em todas as teorias utilizadas nos trabalhos. Teorias menos utilizadas estão contabilizadas na coluna "Outra".

A análise dos artigos também permitiu identificar os termos mais utilizados para designar CC e CV (Tab. 2). Os termos "capacidade colaborativa" e "criação de valor" foram os mais assíduos nos trabalhos, com 67 e 44 aparições, respectivamente. 
Tabela 2 - Nomenclaturas para CC e CV

\begin{tabular}{|c|c|c|c|c|c|}
\hline \multicolumn{2}{|c|}{ Nomenclaturas adotadas } & \multirow{2}{*}{$\begin{array}{c}\text { Capacidade } \\
\text { Colaborativa }\end{array}$} & \multirow{2}{*}{$\begin{array}{c}\begin{array}{c}\text { Criação } \\
\text { de Valor }\end{array} \\
0\end{array}$} & \multirow{2}{*}{$\begin{array}{c}\text { Capacidade } \\
\text { Colaborativa } \\
\text { e Criação de } \\
\text { Valor }\end{array}$} & \multirow{2}{*}{$\begin{array}{c}\text { Total } \\
67\end{array}$} \\
\hline \multirow{4}{*}{$\begin{array}{l}\text { Capacidade } \\
\text { Colaborativa }\end{array}$} & Capacidade Colaborativa & & & & \\
\hline & Capacidade de aliança & 0 & 0 & 1 & 1 \\
\hline & $\begin{array}{l}\text { Capacidade para } \\
\text { integração }\end{array}$ & 1 & 0 & 0 & 1 \\
\hline & $\begin{array}{c}\text { Habilidade para } \\
\text { construção de alianças }\end{array}$ & 1 & 0 & 0 & 1 \\
\hline \multirow{3}{*}{ Criação de Valor } & Criação de Valor & 0 & 3 & 41 & 44 \\
\hline & Ganho relacional & 0 & 0 & 1 & 1 \\
\hline & Valor relacional & 0 & 0 & 1 & 1 \\
\hline
\end{tabular}

\subsection{Atributos de CC e de CV}

Através da leitura dos 73 artigos, foram identificados os atributos utilizados pelos autores que influenciam no desenvolvimento da CC e da CV (Quadros 2 e 3). Primeiramente foram identificados os atributos considerados pelos autores e, em seguida, esses atributos foram agrupados e denominados com um termo principal. Assim, quando as empresas estão dispostas a desenvolverem CC, alguns atributos são necessários, conforme se verifica a seguir.

a) Alinhamento: envolve congruência de metas, sincronização de decisões, monitoramento mútuo, processos, incentivos, especificidade do processo de negócios, dimensão estrutural e cognitiva, além da coordenação do portfólio de alianças (Quadro 2). A congruência de metas é o alinhamento entre objetivos próprios da empresa e os objetivos dos parceiros (CAO; ZHANG, 2011). A sincronização de decisões é uma forma de determinar a maneira mais eficiente e eficaz de usar os recursos disponíveis (SARKAR; AULAKH; MADHOK, 2009). A fim de evitar comportamentos oportunistas, acontece o monitoramento mútuo, no qual ocorre a equidade na observação entre as atividades executadas na parceria por cada membro (LI; ZHENG; ZHUANG, 2017). O alinhamento de processos é a integração de atividades ou da rotina de operações realizadas entre uma firma e seus parceiros (SARAF; LANGDON; GOSAIN, 2007; SUBRAMANI, 2004). Há, também, o alinhamento de incentivos, ou seja, o processo de compartilhamento de custos, de riscos e de benefícios, bem como a formulação de esquemas de incentivos (CAO; ZHANG, 2011). Portanto, considera- 
se necessário para o alinhamento entre as empresas, a especificidade do processo de negócio, ou seja, que as firmas desenvolvam rotinas de procedimentos que auxiliem na eficiência da interação entre os membros (SUBRAMANI, 2004). 
Quadro 2 - Atributos da CC

\begin{tabular}{|c|c|c|}
\hline Atributo & Outras denominações & Autores \\
\hline Alinhamento & $\begin{array}{l}\text { Alinhamento de incentivos/ de processos/ de ris- } \\
\text { cos/Compatibilidade/ Congruência de metas/ Con- } \\
\text { junto de objetivos/ Coordenação do portfólio de ali- } \\
\text { anças/ Dimensão cognitiva/Dimensão estrutural/ } \\
\text { Especificidade do processo de negócios/ Interesse } \\
\text { comum/ Monitoramento mútuo/ Mutualidade/ Sin- } \\
\text { cronização de decisões/ Valores compartilhados }\end{array}$ & $\begin{array}{l}\text { Kanter (1994); Sarkar et al (2001); Brinkerhoff (2002); Möller e Törrönen (2003); Subramani (2004); } \\
\text { Saraf, Langdon e Gosain (2007); Sarkar, Aulakh e Madhok (2009); Cheung, Myers e Mentzer } \\
\text { (2010); Cao e Zhang (2011); Romero e Molina (2011); Al-Tabbaa e Ankrah (2016); Barroso-Méndez } \\
\text { et al. (2016); Bliemel, Mccarth e Maine (2016); Dellyanaa, Simatupangb e Dhewantoc (2016); } \\
\text { Hughes-Morgan e Yao (2016); Matinheikki et al. (2016); Son, Kocabasoglu-Hillmer e Roden (2016); } \\
\text { Srai, Harrington e Tiwari (2016); Wang e Chen (2016); Jones et al. (2017); Li, Zheng e Zhuang } \\
\text { (2017); Wulf e Butel (2017); Yan, Yang e Dooley (2017); Yderfält e Roxenhall (2017) }\end{array}$ \\
\hline Aplicação de TI & $\begin{array}{l}\text { Aplicação de sistema de informação/ Interação atra- } \\
\text { vés da (TI)/ Integração virtual }\end{array}$ & $\begin{array}{l}\text { Monge et al. (1998); Saraf, Langdon e Gosain (2007); Wang e Wei (2007); Hughes-Morgan e Yao } \\
\text { (2016); Li, Zheng e Zhuang (2017); }\end{array}$ \\
\hline Aprendizado & $\begin{array}{l}\text { Acesso ao conhecimento e às habilidades comple- } \\
\text { mentares/ Capacidade absortiva }\end{array}$ & $\begin{array}{l}\text { Kanter (1994); Alvarez e Barney (2001); Möller e Törrönen (2003); Matusik e Heeley (2005); Möller } \\
\text { e Svahn (2006); Wu e Cavusgil (2006); Gulati, Lavie e Singh (2009); Molina-Morales e Martínez- } \\
\text { Fernández (2009); Cheung, Myers e Mentzer (2010); Harrison, Bosse e Phillips (2010); Maurer } \\
\text { (2010); Barroso-Méndez et al. (2016); Dellyanaa, Simatupangb e Dhewantoc (2016); Yeh (2016); } \\
\text { Kähkönen et al. (2017) }\end{array}$ \\
\hline $\begin{array}{l}\text { Apropriação } \\
\text { igualitária }\end{array}$ & Equidade/ Justiça distributiva & $\begin{array}{l}\text { Johnson (1999); Harrison, Bosse e Phillips (2010); Maurer (2010); Wagner, Eggert e Lindemann } \\
\text { (2010); Bouncken e Fredrich (2016) }\end{array}$ \\
\hline $\begin{array}{l}\text { Atributos da } \\
\text { rede }\end{array}$ & $\begin{array}{l}\text { Buraco estrutural/ Centralidade da rede/ Competên- } \\
\text { cia da rede/ Densidade da rede/ Dinâmica da rela- } \\
\text { ção interorganizacional/ Dimensão relacional/ Ele- } \\
\text { mentos estruturais da cadeia de suprimentos/ En- } \\
\text { trelaçamento da aliança }\end{array}$ & $\begin{array}{l}\text { Sarkar, Aulakh e Madhok (2009); Stadtler (2009); Swaminathan e Moorman (2009); Hughes-Morgan } \\
\text { e Yao (2016); Matinheikki et al. (2016); Panda (2016); Son, Kocabasoglu-Hillmer e Roden (2016); } \\
\text { Srai, Harrington e Tiwari (2016); Torkkeli et al. (2016); Wang e Chen (2016); Yeh (2016); Li, Zheng } \\
\text { e Zhuang (2017); Mani et al. (2017); Zhang, Li e Li (2017) }\end{array}$ \\
\hline $\begin{array}{l}\text { Atributos orga- } \\
\text { nizacionais }\end{array}$ & $\begin{array}{l}\text { Capacidades da organização/ Diversidade de par- } \\
\text { cerias, do porttólio/ Experiência da empresa em } \\
\text { parcerias/ Idade da empresa/ Imagem da empresa / } \\
\text { Mentalidade do stakeholder/ Nível de experiência / } \\
\text { Portfólio de alianças fortes/ Reputação, Tamanho e } \\
\text { Tipo de empresa }\end{array}$ & $\begin{array}{l}\text { Alvarez e Barney (2001); Matusik e Heeley (2005); Saraf, Langdon e Gosain (2007); Klein e Rai } \\
\text { (2009); Molina-Morales e Martínez-Fernandez (2009); Sarkar, Aulakh e Madhok (2009); Maure } \\
\text { (2010); Barroso-Méndez et al. (2016); Bouncken e Fredrich (2016); Jraisat (2016); Mindruta, Moeen } \\
\text { e Agarwal (2016); Panda (2016); Piening, Salge e Schäfer (2016); Tomlinson e Fai (2016); Wang e } \\
\text { Chen (2016); Hoehn-Weiss, Karim e Lee (2017); Hsiao et al. (2017); Mani et al. (2017); Pangarkar, } \\
\text { Yuan e Hussain (2017); Rühli et al. (2017); Sadovnikova e Pujari (2017) }\end{array}$ \\
\hline $\begin{array}{l}\text { Compartilha- } \\
\text { mento de co- } \\
\text { nhecimento }\end{array}$ & $\begin{array}{l}\text { Compartilhamento de informação, de novas ideias/ } \\
\text { Compartilhamento estratégiç/ Complementaridade/ } \\
\text { Conhecimento cognitivo/ Criação conjunta de co- } \\
\text { nhecimento/ Fluxo de informação/ Fonte externa de } \\
\text { conhecimento/ Intenção de aprendizado/ Intercâm- } \\
\text { bio bilateral de informações/Intercâmbio de conheci- } \\
\text { mento/ Intercâmbio de informaçôes/ Transferência e } \\
\text { roca de conhecimento e informação }\end{array}$ & $\begin{array}{l}\text { Kanter (1994); Dyer e Singh (1998); Monge et al. (1998); Sarkar et al. (2001); Möller e Törrönen } \\
\text { (2003); Matusik e Heeley (2005); Möller e Svahn (2006); Wu e Cavusgil (2006); Saraf, Langdon e } \\
\text { Gosain (2007); Gulati, Lavie e Singh (2009); Klein e Rai (2009); Molina-Morales e Martinnz-Fernán- } \\
\text { dez (2009); Cheung, Myers e Mentzer (2010); Harrison, Bosse e Phillips (2010); Maurer (2010); } \\
\text { Wagner, Eggert e Lindemann (2010); Cao e Zhang (2011); Romero e Molina (2011); Al-Tabbaa e } \\
\text { Ankrah (2016); Barroso-Méndez et al. (2016); Bliemel, Mccarth e Maine (2016); Charterina, Baster- }- \text { (2016) } \\
\text { retxea e Landeta (2016); Dellyanaa, Simatupangb e Dhewantoc (2016); Hughes-Morgan e Yao } \\
\text { (2016); Jraisat (2016); Loebbecke, Van Fenema e Powell (2016); Zhang, Van Donk e Van der Vaart } \\
\text { (2016); Fang, Wang e Chen (2017); Hsiao et al. (2017); Kähkönen et al. (2017); Paula e Da Silva } \\
\text { (2017); Villani, Greco e Phillips (2017); Wulf e Butel (2017) }\end{array}$ \\
\hline
\end{tabular}


Quadro 2 - Atributos da CC

\begin{tabular}{|c|c|c|}
\hline & Outras denominacões & taroc \\
\hline $\begin{array}{l}\text { Compartilha- } \\
\text { mento de recur- } \\
\text { sos }\end{array}$ & $\begin{array}{l}\text { Compartilhamento de habilidades/ de bens e de fi- } \\
\text { nanças, de infraestrutura, de recursos humanos, de } \\
\text { sistemas/ Complementaridade/ Disponibilidade de } \\
\text { recursos/ Investimento em ativos específicos/ Inves- } \\
\text { timentos idiossincráticos/ Transferência de tecnolo- } \\
\text { gia/ Troca de recursos }\end{array}$ & $\begin{array}{l}\text { Kanter (1994); Dyer e Singh (1998); Madhok e Tallman (1998); Monge et al. (1998); Holm, Eriksson } \\
\text { e Johanson (1999); Alvarez e Barney (2001); Sarkar et al. (2001); Brinkerhoff (2002); Moran (2005); } \\
\text { Klein e Rai (2009); Cheung, Myers e Mentzer (2010); Cao e Zhang (2011); Romero e Molina (2011); } \\
\text { Al-Tabbaa e Ankrah (2016); Bliemel, Mccarth e Maine (2016); Charterina, Basterretxea e Landeta } \\
\text { (2016); Dellyanaa, Simatupangb e Dhewantoc (2016); Hughes-Morgan e Yao (2016); Jraisat (2016); } \\
\text { Mindruta, Moeen e Agarwal (2016); Chen, Chen e Wu (2017); Hoehn-Weiss, Karim e Lee (2017); } \\
\text { Jones et al. (2017); Kähkönen et al. (2017); Mani et al. (2017); Soda e Furlotti (2017); Villani, Greco } \\
\text { e Phillips (2017); Weber et al. (2017); Wu, Wang e Chen (2017) }\end{array}$ \\
\hline $\begin{array}{l}\text { Comprometi- } \\
\text { mento }\end{array}$ & $\begin{array}{l}\text { Compartilhamento de responsabilidades/ Compro- } \\
\text { metimento / Compromisso/ Compromisso mútuo/ } \\
\text { Engajamento / Lealdade / Reciprocidade }\end{array}$ & $\begin{array}{l}\text { Holm, Eriksson e Johanson (1999); Johnson (1999); Sarkar et al. (2001); Wu e Cavusgil (2006); } \\
\text { Harrison, Bosse e Phillips (2010); Al-Tabbaa e Ankrah (2016); Barroso-Mendez et al., (2016); } \\
\text { Schleimer e Faems (2016); Chen, Chen e Wu (2017); Villani et al (2017) }\end{array}$ \\
\hline Comunicação & Comunicação colaborativa & $\begin{array}{l}\text { Monge et al. (1998); Möller e Törrönen (2003); Matusik e Heeley (2005); Cao e Zhang (2011); Al- } \\
\text { Tabbaa e Ankrah (2016); Dellyanaa, Simatupangb e Dhewantoc (2016); Jones et al. (2017) }\end{array}$ \\
\hline Confiança & Confiança mútua/ Confiança & $\begin{array}{l}\text { Kanter (1994); Monge et al. (1998); Johnson (1999); Alvarez e Barney (2001); Sarkar et al. (2001); } \\
\text { Brinkerhoff (2002); Klein e Rai (2009); Molina-Morales e Martínez-Fernández (2009); Cheung, } \\
\text { Myers e Mentzer (2010); Harrison, Bosse e Phillips (2010); Maurer (2010); Wagner, Eggert e } \\
\text { Lindemann (2010); Romero e Molina (2011); Al-Tabbaa e Ankrah (2016); Barroso-Méndez et al. } \\
\text { (2016); Bliemel, Mccarth e Maine (2016); Panda (2016); Schleimer e Faems (2016); Chen, Chen e } \\
\text { Wu (2017); Jones et al. (2017); Wu, Wang e Chen (2017) }\end{array}$ \\
\hline Desempenho & Desempenho da firma e da aliança & $\begin{array}{l}\text { Wu e Cavusgil (2006); Klein e Rai (2009); Jraisat (2016); Teller, Alexander e Floh (2016); Wang e } \\
\text { Chen (2016); Zhang, Van Donk e Van der Vaart (2016); Hoehn-Weiss, Karim e Lee (2017); Hsiao } \\
\text { et al. (2017); Sadovnikova e Pujari (2017) }\end{array}$ \\
\hline $\begin{array}{l}\text { Governança re- } \\
\text { lacional }\end{array}$ & Governança da rede & $\begin{array}{l}\text { Dyer e Singh (1998); Wang e Wei (2007); Sarkar, Aulakh e Madhok (2009); Srai, Harrington e Tiwari } \\
\text { (2016); Yeh (2016); Weber et al. (2017); Wulf e Butel (2017) }\end{array}$ \\
\hline Novas parcerias & $\begin{array}{l}\text { Identificação de oportunidades para parceria/ Inicia- } \\
\text { tiva para relações/ Proatividade para parcerias }\end{array}$ & Wu e Cavusgil (2006); Sarkar, Aulakh e Madhok (2009) \\
\hline $\begin{array}{l}\text { Relacionamento } \\
\text { longo }\end{array}$ & $\begin{array}{l}\text { Continuação do relacionamento/ Duração do relaci- } \\
\text { onamento }\end{array}$ & Johnson (1999); Gulati et. al. (2009); Maurer (2010); Bouncken e Fredrich (2016); Jraisat (2016) \\
\hline
\end{tabular}


A dimensão estrutural caracteriza as atividades desenvolvidas por cada empresa da rede para que haja gerenciamento da aliança (MATINHEIKKI et al., 2016). O gerenciamento, por sua vez, associa-se à coordenação do portfólio de alianças, já que esta é resultante da interação simultânea entre parceiros, a fim de que haja compartilhamento de conhecimentos e estratégias (SARKAR; AULAKH; MADHOK, 2009).

b) Aplicação de TI (Tecnologia da Informação): é importante para a sincronização entre membros. A partir dela, ocorre a integração virtual ou interação através de TI dos parceiros (SARAF; LANGDON; GOSAIN, 2007). Esta permite a união dos sistemas de informação, evitando comportamentos oportunistas, ocasionados pela assimetria de informações (LI; ZHENG; ZHUANG, 2017; SARAF; LANGDON; GOSAIN, 2007; WANG; WEI, 2007).

c) Aprendizado: refere-se à capacidade em absorver o conhecimento compartilhado (capacidade absortiva) (HARRISON; BOSSE; PHILLIPS, 2010; MATUSIK; HEELEY, 2005). Portanto, é a assimilação e o uso das informações que fluem através dos membros da rede de modo que se crie valor para a aliança.

d) Apropriação igualitária de valor: é a justiça distributiva, ou seja, todos os parceiros podem beneficiar-se proporcionalmente aos ganhos gerados pela colaboração (WAGNER; EGGERT; LINDEMANN, 2010).

e) Atributos da rede: são a centralidade, a competência, a dimensão relacional, a densidade, os elementos estruturais, bem como o buraco estrutural e o entrelaçamento da aliança (Quadro 2). As empresas com localização central em uma rede estão expostas a recursos externos mais ricos e têm maior controle e flexibilidade de alocação destes para alcançar seus objetivos (HUGHES-MORGAN; YAO, 2016). A competência da rede é a habilidade que os parceiros têm para desenvolver e manter os laços entre os membros da aliança (TORKKELI et al., 2016). A dimensão relacional é o modo como os laços são estabelecidos entre os membros (PANDA, 2016). Quanto mais intensas e colaborativas forem as interações, maior será a densidade e o fortalecimento da rede (LI; ZHENG; ZHUANG, 2017). O modo como os laços são construídos dependem de elementos estruturais da rede, já que estes determinam o número de laços em alianças e a função de cada empresa no negócio (SUBRAMANI, 2004). O buraco estrutural ocorre em alianças multipartidárias quando uma empresa focal tem a função de ponte para a formação de laços entre outras (MANI et al., 2017). Assim, surgem oportunidades para que a parte conectada se beneficie de informações 
não redundantes fornecidas por ambas as partes. Em cadeias de suprimentos, o número de laços entre as empresas corresponde a um elemento estrutural da rede e influencia a função de cada firma dentro da cadeia (STADTLER, 2009). Logo, a empresa com mais pontes terá retornos ponderados mais altos do que seu parceiro (HUGHES-MORGAN; YAO, 2016).

f) Atributos organizacionais: tamanho, idade, tipo e reputação da empresa, bem como a experiência em parcerias, a mentalidade do stakeholder, o portfólio de alianças fortes, a diversidade no portfólio de alianças e as capacidades da organização formam esses atributos. Para Hsiao et al. (2017), dois fatores devem ser levados em conta na parceria: o tamanho e a idade da empresa. O primeiro aspecto pode influenciar o desempenho, isto é, a força competitiva extraída da colaboração (WU; CAVUSGIL, 2006), e o segundo está ligado ao nível de experiência. Além desses, o tipo de empresa também é importante (MOLINA-MORALES; MARTÍNEZFERNÁNDEZ, 2009). Não obstante, a reputação ou imagem no mercado deve ser levada em consideração, pois as organizações com reputação positiva são caracterizadas como de baixos riscos relacionais (MANI et al., 2017). A imagem está relacionada às capacidades da organização, ou seja, às habilidades possuídas pela firma e oriundas das práticas desenvolvidas em seus diversos âmbitos, como marketing, tecnologia e processos (SADOVNIKOVA; PUJARI, 2017). As empresas com mais experiência em alianças apresentam melhores habilidades no engajamento em novas colaborações e aumentam as chances de captura de valor (BOUNCKEN; FREDRICH, 2016; PANGARKAR; YUAN; HUSSAIN, 2017). Assim, o conjunto de expectativas, de percepções e de atitudes do membro da aliança aumenta quando da participação de uma parceria (RÜHLI et al., 2017). Um portfólio de alianças fortes garante vantagem competitiva (SARKAR; AULAKH; MADHOK, 2009). A diversidade no portfólio de alianças e de atributos organizacionais, como a estrutura hierárquica, o estilo operacional e os sistemas para a execução de tarefas de cada empresa também garantem vantagens à rede (PANDA, 2016; SARKAR; AULAKH; MADHOK, 2009). As capacidades da organização são igualmente relevantes em uma colaboração, já que através delas são estabelecidas as habilidades gerais de empresas, através de seus diversos departamentos, como Marketing e Pesquisa \& Desenvolvimento (SADOVNIKOVA; PUJARI, 2017). 
Quadro 3 - Atributos da CV

\begin{tabular}{|c|c|c|}
\hline Atributo & Outras denominações & Autores \\
\hline Capacidades & $\begin{array}{l}\text { Capacidades de lidar com situações inesperadas/ Desenvolvimento de } \\
\text { capacidades/ Resposta em tempo reduzido/ Resposta pragmática }\end{array}$ & Johnson (1999); Brinkerhoff (2002); Harrison, Bosse e Phillips (2010); Zhang, Li e Li (2017) \\
\hline Conhecimento & Especificidade do domínio do conhecimento & Subramani (2004); Volschenk, Ungerer e Smit (2016); Saunila et al. (2017) \\
\hline Demanda & Aumento da demanda/ Aumento das vendas/ Maior produtividade & $\begin{array}{l}\text { Möller e Törrönen (2003); Klein e Rai (2009); Harrison, Bosse e Phillips (2010); Cao e Zhang } \\
\text { (2011); Jraisat (2016); Tomlinson e Fai (2016) }\end{array}$ \\
\hline Eficiência & Eficiência do processo/ no serviço/ Transação simplificada e eficiente & $\begin{array}{l}\text { Johnson (1999); Möller e Törrönen (2003); Subramani (2004); Gulati, Lavie e Singh (2009); } \\
\text { Harrison, Bosse e Phillips (2010); Cao e Zhang (2011); Bliemel, Mccarth e Maine (2016); } \\
\text { Dellyanaa, Simatupangb e Dhewantoc (2016); Jraisat (2016); Jones et al. (2017) }\end{array}$ \\
\hline Flexibilidade & Flexibilidade de recursos/ Redução do tempo de ciclo de inovação & $\begin{array}{l}\text { Johnson (1999); Brinkerhoff (2002); Möller e Törrönen (2003); Wang e Wei (2007); Klein e Rai } \\
\text { (2009); Cao e Zhang (2011); Romero e Molina (2011); Hughes-Morgan e Yao (2016); Hsiao } \\
\text { et al. (2017); Kähkönen et al. (2017) }\end{array}$ \\
\hline $\begin{array}{l}\text { Gestão da } \\
\text { Qualidade }\end{array}$ & $\begin{array}{l}\text { Qualidade/ Qualidade da informação/ Qualidade do recurso humano/ } \\
\text { Qualidade do relacionamento }\end{array}$ & $\begin{array}{l}\text { Madhok e Tallman (1998); Monge et al. (1998); Cao e Zhang (2011); Charterina, Basterretxea } \\
\text { e Landeta (2016); Dellyanaa, Simatupangb e Dhewantoc (2016); Jraisat (2016); Hsiao et al. } \\
\text { (2017) }\end{array}$ \\
\hline $\begin{array}{l}\text { Histórico de } \\
\text { parcerias }\end{array}$ & Histórico de parcerias & Gulati, Lavie e Singh (2009) \\
\hline Investimento & $\begin{array}{l}\text { Aumento anormal de retornos/ Aumento da ROI/ Efeito positivo no re- } \\
\text { torno sobre patrimônio/ Retornos acima do esperado }\end{array}$ & $\begin{array}{l}\text { Swaminathan e Moorman (2009); Cao e Zhang (2011); Bouncken e Fredrich (2016); Hughes- } \\
\text { Morgan e Yao (2016); Sadovnikova e Pujari (2017) }\end{array}$ \\
\hline Lucro & Aumento da margem de lucro/ Rentabilidade/ Vantagem financeira & $\begin{array}{l}\text { Monge et al. (1998); Johnson (1999); Möller e Törrönen (2003); Matusik e Heeley (2005); Cao } \\
\text { e Zhang (2011); Bliemel, Mccarth e Maine (2016); Dellyanaa, Simatupangb e Dhewantoc } \\
\text { (2016); Saunila et al. (2017); Jraisat (2016); Torkkeli et al. (2016); Zhang, Li e Li (2017) }\end{array}$ \\
\hline $\begin{array}{l}\text { Planejamento e } \\
\text { Gestão }\end{array}$ & $\begin{array}{l}\text { Criar capacidade organizacional/ Maior controle dos recursos/ Melhor } \\
\text { planejamento/ Melhoria da gestão de ativos/ Redução de custos/ Susten- } \\
\text { tabilidade do negócio/ Vantagens na gestão da logística }\end{array}$ & $\begin{array}{l}\text { Johnson (1999); Subramani (2004); Klein e Rai (2009); Jraisat (2016); Jones et al. (2017); } \\
\text { Saunila et al. (2017) }\end{array}$ \\
\hline \multicolumn{2}{|c|}{ Reputação da rede } & Swaminathan e Moorman (2009); Saunila et al. (2017) \\
\hline Satisfação & Satisfação do cliente/ no desenvolvimento de projetos/ relacional & Monge et al. (1998); Wagner, Eggert e Lindemann (2010); Jraisat (2016); Saunila et al. (2017) \\
\hline Sinergia & Sinergia empresarial & $\begin{array}{l}\text { Kanter (1994); Dyer e Singh (1998); Madhok e Tallman (1998); Holm, Eriksson e Johanson } \\
\text { (1999); Cao e Zhang (2011); Romero e Molina (2011); Bliemel, Mccarth e Maine (2016); Char- } \\
\text { terina, Basterretxea e Landeta (2016) }\end{array}$ \\
\hline $\begin{array}{l}\text { Tecnologia e } \\
\text { Inovação }\end{array}$ & $\begin{array}{l}\text { Desempenho inovativo/ Desenvolvimento de novos produtos/ Fortaleci- } \\
\text { mento do desenvolvimento tecnológico/ Inovação do produto/ Know-how } \\
\text { tecnológico/ }\end{array}$ & $\begin{array}{l}\text { Johnson (1999); Walter, Ritter e Gemünden (2001); Brinkerhoff (2002); Möller e Törrönen } \\
\text { (2003); Moran (2005); Klein e Rai (2009); Molina-Morales e Martínez-Fernández (2009); Har- } \\
\text { rison, Bosse e Phillips (2010); Maurer (2010); Cao e Zhang (2011); Romero e Molina (2011); } \\
\text { Charterina, Basterretxea e Landeta (2016); Dellyanaa, Simatupangb e Dhewantoc (2016); } \\
\text { Hughes-Morgan e Yao (2016); Piening, Salge e Schäfer (2016); Srai, Harrington e Tiwari } \\
\text { (2016); Tomlinson e Fai (2016); Fang, Wang e Chen (2017); Hsiao et al. (2017); Kähkönen et } \\
\text { al. (2017); Paula e Da Silva (2017); Sadovnikova e Pujari (2017); Wu, Wang e Chen (2017); } \\
\text { Yan, Yang e Dooley (2017) }\end{array}$ \\
\hline
\end{tabular}

Revista Produção Online. Florianópolis, SC, v. 19, n. 4, p. 1146-1176, 2019 
g) Compartilhamento de informações: é a extensão dos conhecimentos possuídos por uma empresa acerca de capacidades específicas, know how e informações sobre o mercado para além de suas fronteiras (LOEBBECKE; VAN FENEMA; POWELL, 2016; SARAF; LANGDON; GOSAIN, 2007).

h) Compartilhamento de recurso: refere-se ao processo de alavancar e investir em capacidades e ativos com parceiros da rede (CAO; ZHANG, 2011).

i) Comprometimento organizacional: é uma maneira de reduzir comportamentos oportunistas (WU; CAVUSGIL, 2006). Quando os parceiros estão comprometidos, eles trabalharão para o sucesso da parceria, refletindo na cooperação e no aprendizado (BARROSO-MÉNDEZ et al., 2016).

j) Comunicação colaborativa: é o contato e o processo de transmissão de mensagens entre os parceiros em termos de frequência, de direção, de modo e de influência estratégica (CAO; ZHANG, 2011).

k) Confiança: torna os parceiros mais propensos a compartilharem informações importantes e a manterem um diálogo construtivo. A confiança afeta a cooperação, a aprendizagem do relacionamento de forma direta e positiva (ALVAREZ; BARNEY, 2001; BARROSO-MÉNDEZ et al., 2016; BRINKERHOFF, 2002; CHEUNG; MYERS; MENTZER, 2010; HARRISON; BOSSE; PHILLIPS, 2010; JOHNSON, 1999; PANDA, 2016) e eleva as possibilidades de colaborações futuras (WAGNER; EGGERT; LINDEMANN, 2010).

I) Desempenho da firma: refere-se às várias medidas de atividades da empresa para avaliar seu nível de realizações, que fornecem a informação necessária para que os tomadores de decisão planejem, controlem e direcionem suas atividades em relação aos relacionamentos colaborativos atuais e futuros (JRAISAT, 2016).

m) Governança relacional: é estabelecida através de rotinas comportamentais para que os ativos especializados da aliança e das empresas estejam protegidos (DYER; SINGH, 1998; SARKAR; AULAKH; MADHOK, 2009; WANG; WEI, 2007).

n) Novas parcerias: são estabelecidas pela identificação de oportunidades, corresponde à habilidade de analisar o mercado e de constatar a existência de candidatos com recursos complementares (WU; CAVUSGIL, 2006). Em seguida, ocorre o engajamento para a formação da rede antes dos competidores, situação característica da pro atividade para parcerias (SARKAR; AULAKH; MADHOK, 2009). 
o) Relacionamento longo: com os mesmos parceiros oferece melhores benefícios do que as experiências anteriores com parceiros diferentes, pois a integração é mais estratégica (GULATI; LAVIE; SINGH, 2009; JOHNSON, 1999).

A partir do desenvolvimento de $\mathrm{CC}$, as empresas obtêm diversos benefícios (CHEUNG; MYERS; MENTZER, 2010), uma vez que os parceiros trazem recursos únicos e valiosos de modo a potencializarem a CV (SARKAR et al., 2001). Dessa forma, foram identificados quatorze benefícios que geram $\mathrm{CV}$ :

a) Capacidades: o desenvolvimento de capacidades é considerado um importante ganho relacional, pois as habilidades desenvolvidas pela interação com outros membros da aliança influenciam na obtenção de vantagem competitiva sustentável por parte de uma firma focal (ZHANG; LI; LI, 2017).

b) Conhecimento: a CV é resultado do fluxo de informações que auxiliam no desenvolvimento conjunto dos membros da aliança (SAUNILA et al., 2017; VOLSCHENK; UNGERER; SMIT, 2016). A especificidade do domínio do conhecimento é o benefício oriundo da obtenção de informações facilitam o planejamento e a execução de tarefas por parte dos membros (SUBRAMANI, 2004).

c) Demanda: se a demanda das empresas aumenta em função dos relacionamentos, ocorre um crescimento das vendas, e as firmas têm maior produtividade (CAO; ZHANG, 2011; HARRISON; BOSSE; PHILLIPS, 2010; KLEIN; RAI, 2009).

d) Eficiência: ganho relacional que beneficia as empresas pela simplificação de procedimentos, de maneira a haver melhorias na oferta, na execução de serviços e no desenvolvimento de processos (JONES et al., 2017; SUBRAMANI, 2004).

e) Flexibilidade: pode ser gerada em função da colaboração e está relacionada às mudanças nas ofertas de produto ou de serviço em resposta às necessidades do cliente (CAO; ZHANG, 2011).

f) Gestão da qualidade: envolve, a partir da colaboração, respostas mais rápidas às necessidades dos clientes com produtos de alta qualidade e de design inovador, bem como excelente serviço pós-venda (CAO; ZHANG, 2011).

g) Histórico de parcerias: influencia no sucesso das novas alianças. Assim, os tomadores de decisão precisam ser criteriosos no gerenciamento das alianças, pois suas escolhas orientarão as demais e influenciarão nos ganhos (GULATI; LAVIE; SINGH, 2009). 
h) Investimento: é considerado como uma vantagem da colaboração a partir do momento em que há retornos acima do esperado nas aplicações, obtendo-se um efeito positivo no retorno sobre o patrimônio (CAO; ZHANG, 2011; SWAMINATHAN; MOORMAN, 2009) .

i) Lucro: é considerado uma vantagem financeira; ligado ao aumento da margem de lucro e à rentabilidade (BLIEMEL; MCCARTHY; MAINE, 2016; CAO; ZHANG, 2011; DELLYANAA; SIMATUPANGB; DHEWANTOC, 2016; JOHNSON, 1999; JRAISAT, 2016; MATUSIK; HEELEY, 2005; MÖLLER; TÖRRÖNEN, 2003; MONGE et al., 1998; SAUNILA et al., 2017; TORKKELI et al., 2016; ZHANG; LI; LI, 2017).

j) Planejamento e gestão: acarretam melhorias para os parceiros de uma aliança através de vantagens como: maior controle dos recursos, melhoria na gestão de ativos e de logística, redução de custos, criação de capacidades organizacionais e sustentabilidade do negócio (SAUNILA et al., 2017).

k) Reputação: influencia no processo de $\mathrm{CV}$, pois pressiona os membros de uma relação a manter sinais de qualidade na colaboração (SWAMINATHAN; MOORMAN, 2009).

I) Satisfação: aborda três tipos: desenvolvimento de projetos, relacional e cliente. Os valores criados pelo trabalho conjunto e que influencia positivamente na formação de novas colaborações estão relacionados à satisfação no desenvolvimento de projetos (WAGNER; EGGERT; LINDEMANN, 2010). A constatação dos benefícios adquiridos através da participação na aliança está ligada à satisfação relacional (WAGNER; EGGERT; LINDEMANN, 2010). E, a satisfação do cliente é um capital a ser mantido para a manutenção do lucro da empresa (SAUNILA et al., 2017).

m) Sinergia empresarial: ocorre quando os ganhos obtidos pela parceria são maiores do que os individuais devido à combinação de recursos complementares e compatíveis entre os parceiros (CAO; ZHANG, 2011).

n) Tecnologia e inovação: são benefícios garantidos pelos conhecimentos adquiridos na parceria (HSIAO et al., 2017). A inovação refere-se à medida em que uma empresa trabalha em conjunto com os parceiros na introdução de novos processos, produtos ou serviços (CAO; ZHANG, 2011). 


\section{DISCUSSÃO}

A CV proveniente da parceria está relacionada com a propensão das empresas em formar parcerias, o que é essencial para o sucesso do relacionamento. Dessa forma, após extrair da literatura tanto os atributos de CC quanto de CV, foi possível constatar que alguns atributos de CC influenciam no processo de CV conjunto (Quadro 4). Por meio da análise de conteúdo dos artigos, também foi possível identificar tendências de pesquisas sobre CC e CV no contexto de relacionamento interorganizacionais, como por exemplo:

- Embora Dyer seja o autor mais citado, assim como a sua obra, as teorias mais utilizadas como plano de fundo são a VBR e a TCT. Isso demonstra que os autores partem do princípio que a CC que gera CV, deve, consequentemente, impulsionar o desenvolvimento de rendas relacionais.

- Quando se trata de pesquisas cujo enfoque é a CC e a CV de forma conjunta, o survey é o método mais adotado; os relacionamentos mais estudados são redes, alianças estratégicas e cadeias de suprimentos.

- Embora utilizem diferentes termos para CC, o mais adotado é, de fato, capacidade colaborativa, assim como ocorre para o termo CV (sendo este o mais presente nas pesquisas analisadas).

A pesquisa apresentada neste artigo também permitiu identificar gaps e oportunidades para futuras pesquisas (Fig.4), conforme se verifica a seguir.

a) Métodos: Pesquisas futuras podem explorar a adoção de modelagem matemática para analisar a influência dos fatores de CC sobre os de CV por meio de metodologias multicritério. As pesquisas conceituais também são escassas, sugerindo o desenvolvimento de outras RSL. Embora o estudo de caso tenha sido abordado, ele ainda é em menor número quando comparado ao survey. Dessa forma, futuras pesquisas podem adotar esse método para explorar em profundidade a temática e em conjunto com outros métodos (métodos mistos).

b) Tipos de arranjos: Pesquisas futuras podem explorar o potencial de desenvolvimento de $\mathrm{CC}$ e CV em outros tipos de relacionamentos interorganizacionais, além de cadeias de suprimentos ou alianças. Por exemplo, em função da ampliação das pressões econômicas, sociais e políticas, outros tipos de colaboração podem ocorrer 
para lidar com problemas sociais complexos, como aquelas que envolvem empresas, universidades e organizações sem fins lucrativos. 


\begin{tabular}{|c|c|c|c|}
\hline & & & \\
\hline Alinhamento & $\begin{array}{c}\text { Histórico da } \\
\text { parceria }\end{array}$ & $\begin{array}{l}\text { O sucesso do alinhamento (congruência de metas, } \\
\text { sincronização de decisões, monitoramento mútuo, } \\
\text { processos, incentivos, especificidade do processo } \\
\text { de negócios, dimensão estrutural e cognitiva, co- } \\
\text { ordenação do portfólio de alianças) entre as em- } \\
\text { presas influenciará positivamente o histórico da } \\
\text { parceria. }\end{array}$ & $\begin{array}{l}\text { Kanter (1994); Sarkar et al. (2001); Brinkerhoff (2002); Möller e Törrönen (2003); } \\
\text { Subramani (2004); Saraf, Langdon e Gosain (2007); Gulati, Lavie e Singh (2009); } \\
\text { Sarkar, Aulakh e Madhok (2009); Cheung, Myers e Mentzer (2010); Cao e Zhang } \\
\text { (2011); Romero e Molina (2011); Al-Tabbaa e Ankrah (2016); Barroso-Méndez et } \\
\text { al. (2016); Bliemel, Mccarth e Maine (2016); Dellyanaa, Simatupangb e Dhewantoc } \\
\text { (2016); Hughes-Morgan e Yao (2016); Matinheikki et al. (2016); Son, Kocabasoglu- } \\
\text { Hillmer e Roden (2016); Srai, Harrington e Tiwari (2016); Wang e Chen (2016); } \\
\text { Jones et al. (2017); Li, Zheng e Zhuang (2017); Wulf e Butel (2017); Yan, Yang e } \\
\text { Dooley (2017); Yderfält \& Roxenhall (2017) }\end{array}$ \\
\hline Aprendizado & $\begin{array}{l}\text { Capacidades, } \\
\text { Conheci- } \\
\text { mento, Tecno- } \\
\text { logia e Inova- } \\
\quad \text { ção }\end{array}$ & $\begin{array}{l}\text { Através do aprendizado, é possível que uma firma } \\
\text { focal desenvolva capacidades que antes não eram } \\
\text { possuídas, sejam ligadas à especificidade do do- } \\
\text { mínio do conhecimento ou à tecnologia e inovação. }\end{array}$ & $\begin{array}{l}\text { Kanter (1994); Johnson (1999); Alvarez e Barney (2001); Walter, Ritter e } \\
\text { Gemünden (2001); Brinkerhoff (2002); Möller e Törrönen (2003); Subramani } \\
\text { (2004); Matusik e Heeley (2005); Moran (2005); Möller e Svahn (2006); Wu e Ca- } \\
\text { vusgil (2006); Gulati, Lavie e Singh (2009); Klein e Rai (2009); Molina-Morales e } \\
\text { Martínez-Fernández (2009); Cheung, Myers e Mentzer (2010); Harrison, Bosse e } \\
\text { Phillips (2010); Maurer (2010); Cao e Zhang (2011); Romero e Molina (2011); Bar- } \\
\text { roso-Méndez et al. (2016); Charterina, Basterretxea e Landeta (2016); Dellyanaa, } \\
\text { Simatupangb e Dhewantoc (2016); Hughes-Morgan e Yao (2016); Piening, Salge } \\
\text { e Schäfer (2016); Srai, Harrington e Tiwari (2016); Tomlinson e Fai (2016); Vols- } \\
\text { chenk, Ungerer e Smit (2016); Yeh (2016); Fang, Wang e Chen (2017); Hsiao et } \\
\text { al. (2017); Kähkönen et al. (2017); Paula e Da Silva (2017); Sadovnikova e Pujari } \\
\text { (2017); Saunila et al. (2017); Wu, Wang e Chen (2017); Yan, Yang e Dooley (2017); } \\
\text { Zhang, Li e Li (2017) }\end{array}$ \\
\hline $\begin{array}{l}\text { Atributos da } \\
\text { rede }\end{array}$ & $\begin{array}{c}\text { Histórico da } \\
\text { parceria }\end{array}$ & $\begin{array}{l}\text { A competência da rede é a habilidade que os par- } \\
\text { ceiros têm para desenvolver e manter os laços en- } \\
\text { tre os membros da aliança, situação que reflete no } \\
\text { histórico da parceria. }\end{array}$ & $\begin{array}{l}\text { Gulati, Lavie e Singh (2009); Hughes-Morgan e Yao (2016); Li, Zheng e Zhuang } \\
\text { (2017); Mani et al. (2017); Matinheikki et al. (2016); Panda (2016); Sarkar, Aulakh } \\
\text { e Madhok (2009); Son, Kocabasoglu-Hillmer e Roden (2016); Srai, Harrington e } \\
\text { Tiwari (2016); Stadtler (2009); Swaminathan e Moorman (2009); Torkkeli et al. } \\
\text { (2016); Wang e Chen (2016); Yeh (2016); Zhang, Li e Li (2017) }\end{array}$ \\
\hline
\end{tabular}




\begin{tabular}{|c|c|c|c|}
\hline CC & CV & Por que? & Autores \\
\hline $\begin{array}{l}\text { Atributos or- } \\
\text { ganizacio- } \\
\text { nais }\end{array}$ & $\begin{array}{c}\text { Capacidades, } \\
\text { Conheci- } \\
\text { mento, De- } \\
\text { manda, Efici- } \\
\text { ência, Flexibili- } \\
\text { dade, Gestão } \\
\text { da qualidade, } \\
\text { Lucro, Plane- } \\
\text { jamento e ges- } \\
\text { tão }\end{array}$ & $\begin{array}{l}\text { Os atributos de uma organização influenciam no } \\
\text { modo como ocorrerá a interação entre os membros } \\
\text { e, por consequência, quais serão os ganhos rela- } \\
\text { cionais. }\end{array}$ & $\begin{array}{l}\text { Monge et al. (1998); Johnson (1999); Alvarez e Barney (2001); Brinkerhoff (2002); } \\
\text { Möller e Törrönen (2003); Subramani (2004); Matusik e Heeley (2005); Saraf, } \\
\text { Langdon e Gosain (2007); Wang e Wei (2007); Gulati, Lavie e Singh (2009); Klein } \\
\text { e Rai (2009); Molina-Morales e Martínez-Fernández (2009); Sarkar, Aulakh e Mad- } \\
\text { hok (2009); Harrison, Bosse e Phillips (2010); Maurer (2010); Cao e Zhang (2011); } \\
\text { Romero e Molina (2011); Barroso-Méndez et al. (2016); Bliemel, Mccarth e Maine } \\
\text { (2016); Bouncken e Fredrich (2016); Dellyanaa, Simatupangb e Dhewantoc (2016); } \\
\text { Hughes-Morgan e Yao (2016); Jraisat (2016); Mindruta, Moeen e Agarwal (2016); } \\
\text { Panda (2016); Piening, Salge e Schäfer (2016); Tomlinson e Fai (2016); Torkkeli } \\
\text { et al. (2016); Volschenk, Ungerer e Smit (2016); Wang e Chen (2016); Hoehn- } \\
\text { Weiss, Karim e Lee (2017); Hsiao et al. (2017); Jones et al. (2017); Kähkönen et } \\
\text { al. (2017); Mani et al. (2017); Pangarkar, Yuan e Hussain (2017); Rühli et al. (2017); } \\
\text { Sadovnikova e Pujari (2017); Saunila et al. (2017); Zhang, Li e Li (2017) }\end{array}$ \\
\hline $\begin{array}{l}\text { Compartilha- } \\
\text { mento de co- } \\
\text { nhecimen-to }\end{array}$ & Conhecimento & $\begin{array}{l}\text { O conhecimento compartilhado promove, através } \\
\text { da especificidade do domínio do conhecimento, a } \\
\text { facilitação do planejamento e da execução dos ne- } \\
\text { gócios. }\end{array}$ & $\begin{array}{l}\text { Kanter (1994); Dyer e Singh (1998); Monge et al. (1998); Sarkar et al. (2001); Möller } \\
\text { e Törrönen (2003); Subramani (2004); Matusik e Heeley (2005); Möller e Svahn } \\
\text { (2006); Wu e Cavusgil (2006); Saraf, Langdon e Gosain (2007); Gulati, Lavie e } \\
\text { Singh (2009); Klein e Rai (2009); Molina-Morales e Martínez-Fernández (2009); } \\
\text { Cheung, Myers e Mentzer (2010); Harrison, Bosse e Phillips (2010); Maurer (2010); } \\
\text { Wagner, Eggert e Lindemann (2010); Cao e Zhang (2011); Romero e Molina } \\
\text { (2011); Al-Tabbaa e Ankrah (2016); Barroso-Méndez et al. (2016); Bliemel, Mccarth } \\
\text { e Maine (2016); Charterina, Basterretxea e Landeta (2016); Dellyanaa, Simatu- } \\
\text { pangb e Dhewantoc (2016); Hughes-Morgan e Yao (2016); Jraisat (2016); } \\
\text { Loebbecke, Van Fenema e Powell (2016); Volschenk, Ungerer e Smit (2016); } \\
\text { Zhang, Van Donk e Van der Vaart (2016); Fang, Wang e Chen (2017); Hsiao et al. } \\
\text { (2017); Kähkönen et al. (2017); Paula e Da Silva (2017); Saunila et al. (2017); Vil- } \\
\text { lani, Greco e Phillips (2017); Wulf e Butel (2017) }\end{array}$ \\
\hline
\end{tabular}




\begin{tabular}{|c|c|c|c|}
\hline $\mathrm{CC}$ & CV & Por que? & Autores \\
\hline $\begin{array}{l}\text { Compartilha- } \\
\text { mento de re- } \\
\text { cursos }\end{array}$ & $\begin{array}{l}\text { Capacidades, } \\
\text { Conhecimen- } \\
\text { to, Investi- } \\
\text { mento, Siner- } \\
\text { gia, Tecnolo- } \\
\text { gia e Inovação }\end{array}$ & $\begin{array}{l}\text { O compartilhamento de recursos resulta na gera- } \\
\text { ção de capacidades. O desenvolvimento de novas } \\
\text { tecnologias ou inovação também é reflexo dos co- } \\
\text { nhecimentos compartilhados. A partir disso, as fir- } \\
\text { mas tornam-se mais aptas a compreender o mer- } \\
\text { cado e a alavancar seus ganhos financeiros coleti- } \\
\text { vamente. }\end{array}$ & $\begin{array}{l}\text { Kanter (1994); Dyer e Singh (1998); Madhok e Tallman (1998); Monge et al. (1998); } \\
\text { Holm, Eriksson e Johanson (1999); Johnson (1999); Alvarez e Barney (2001); } \\
\text { Sarkar et al. (2001); alter, Ritter e Gemünden (2001); Brinkerhoff (2002); Möller e } \\
\text { Törrönen (2003); Subramani (2004); Moran (2005); Klein e Rai (2009); Molina-Mo- } \\
\text { rales e Martínez-Fernández (2009); Swaminathan e Moorman (2009); Cheung, My- } \\
\text { ers e Mentzer (2010); Harrison, Bosse e Phillips (2010); Maurer (2010); Cao e } \\
\text { Zhang (2011); Romero e Molina (2011); Al-Tabbaa e Ankrah (2016); Bliemel, } \\
\text { Mccarth e Maine (2016); Bouncken e Fredrich (2016); Charterina, Basterretxea e } \\
\text { Landeta (2016); Dellyanaa, Simatupangb e Dhewantoc (2016); Hughes-Morgan e } \\
\text { Yao (2016); Jraisat (2016); Mindruta, Moeen e Agarwal (2016); Piening, Salge e } \\
\text { Schäfer (2016); Srai, Harrington e Tiwari (2016); Tomlinson e Fai (2016); Vol- } \\
\text { schenk, Ungerer e Smit (2016); Chen, Chen e Wu (2017); Fang, Wang e Chen } \\
\text { (2017); Hoehn-Weiss, Karim e Lee (2017); Hsiao et al. (2017); Jones et al. (2017); } \\
\text { Kähkönen et al. (2017); Mani et al. (2017); Paula e Da Silva (2017); Sadovnikova } \\
\text { e Pujari (2017); Saunila et al. (2017); Soda e Furlotti (2017); Villani, Greco e Phillips } \\
\text { (2017); W Weber et al. (2017); Wu, Wang e Chen (2017); Yan, Yang e Dooley } \\
\text { (2017); Zhang, Li e Li (2017) }\end{array}$ \\
\hline $\begin{array}{l}\text { Comprometi- } \\
\text { mento }\end{array}$ & $\begin{array}{l}\text { Histórico de } \\
\text { parceria, Re- } \\
\text { putação da } \\
\text { rede, Satisfa- } \\
\quad \text { ção }\end{array}$ & $\begin{array}{l}\text { O comprometimento com os parceiros reflete na } \\
\text { permanência dos membros, fomentando um histó- } \\
\text { rico de relacionamentos interorganizacionais. Por } \\
\text { sua vez, o sucesso dessas relações assegura a } \\
\text { boa reputação da rede e os resultados obtidos } \\
\text { através da aliança resultam em satisfação para os } \\
\text { membros. }\end{array}$ & $\begin{array}{l}\text { Al-Tabbaa e Ankrah (2016); Barroso-Méndez et al., (2016); Chen, Chen e Wu } \\
\text { (2017); Gulati, Lavie e Singh (2009); Harrison, Bosse e Phillips (2010); Holm, Eri- } \\
\text { ksson e Johanson (1999); Johnson (1999); Jraisat (2016); Monge et al. (1998); } \\
\text { Sarkar et al. (2001); Saunila et al. (2017); Schleimer e Faems (2016); Swaminathan } \\
\text { e Moorman (2009); Villani, Greco e Phillips (2017); Wagner, Eggert e Lindemann } \\
\text { (2010); Wu e Cavusgil (2006) }\end{array}$ \\
\hline $\begin{array}{l}\text { Comunica- } \\
\text { ção }\end{array}$ & $\begin{array}{l}\text { Conhecimen- } \\
\text { to, Sinergia }\end{array}$ & $\begin{array}{l}\text { O conhecimento é resultado do fluxo de informa- } \\
\text { ções, obtidos através da comunicação colabora- } \\
\text { tiva, que auxiliam no desenvolvimento conjunto } \\
\text { dos membros da aliança. A partir disso, os ganhos } \\
\text { obtidos pela parceria são maiores do que os indi- } \\
\text { viduais. }\end{array}$ & $\begin{array}{l}\text { Kanter (1994); Dyer e Singh (1998); Madhok e Tallman (1998); Monge et al. (1998); } \\
\text { Holm, Eriksson e Johanson (1999); Möller e Törrönen (2003); Matusik e Heeley } \\
\text { (2005); Cao e Zhang (2011); Romero e Molina (2011); Al-Tabbaa e Ankrah (2016); } \\
\text { Bliemel, Mccarth e Maine (2016); Charterina, Basterretxea e Landeta (2016); Del- } \\
\text { lyanaa, Simatupangb e Dhewantoc (2016); Jones et al. (2017) }\end{array}$ \\
\hline
\end{tabular}




\begin{tabular}{|c|c|c|c|}
\hline CC & Cy & & \\
\hline Confiança & Satisfação & $\begin{array}{l}\text { A confiança afeta a cooperação e a aprendizagem, } \\
\text { esses benefícios adquiridos estão ligados à satis- } \\
\text { fação relacional. Já confiança relacional eleva as } \\
\text { possibilidades de colaborações futuras, isso está } \\
\text { relacionado à satisfação no desenvolvimento de } \\
\text { projetos. }\end{array}$ & $\begin{array}{l}\text { Kanter (1994); Monge et al. (1998); Johnson (1999); Alvarez e Barney (2001); } \\
\text { Sarkar et al. (2001); Brinkerhoff (2002); Klein e Rai (2009); Molina-Morales e Mar- } \\
\text { tínez-Fernández (2009); Cheung, Myers e Mentzer (2010); Harrison, Bosse e Phil- } \\
\text { lips (2010); Maurer (2010); Wagner, Eggert e Lindemann (2010); Romero e Molina } \\
\text { (2011); Al-Tabbaa e Ankrah (2016); Barroso-Méndez et al. (2016); Bliemel, Mccarth } \\
\text { e Maine (2016); Jraisat (2016); Panda (2016); Schleimer e Faems (2016); Chen, } \\
\text { Chen e Wu (2017); Jones et al. (2017); Saunila et al. (2017); Wu, Wang e Chen } \\
\text { (2017) }\end{array}$ \\
\hline Desempenho & Lucro & $\begin{array}{l}\text { O desempenho é um processo pelo qual uma em- } \\
\text { presa usa várias medidas de atividades para ava- } \\
\text { liar seu nível de realizações. }\end{array}$ & $\begin{array}{l}\text { Monge et al. (1998); Johnson (1999); Möller e Törrönen (2003); Matusik e Heeley } \\
\text { (2005); Wu e Cavusgil (2006); Klein e Rai (2009); Cao e Zhang (2011); Bliemel, } \\
\text { Mccarth e Maine (2016); Dellyanaa, Simatupangb e Dhewantoc (2016); Jraisat } \\
\text { (2016); Teller, Alexander e Floh (2016); Torkkeli et al. (2016); Wang e Chen (2016); } \\
\text { Zhang, Van Donk e Van der Vaart (2016); Hoehn-Weiss, Karim e Lee (2017); Hsiao } \\
\text { et al. (2017); Sadovnikova e Pujari (2017); Saunila et al. (2017); bZhang, Li e Li } \\
\text { (2017) }\end{array}$ \\
\hline $\begin{array}{l}\text { Governança } \\
\text { relacional }\end{array}$ & $\begin{array}{l}\text { Histórico de } \\
\text { parceria, Re- } \\
\text { putação da } \\
\text { rede }\end{array}$ & $\begin{array}{l}\text { Ao estabelecer rotinas comportamentais para que } \\
\text { os ativos especializados da aliança e das empre- } \\
\text { sas estejam protegidos, o histórico de parceria e a } \\
\text { reputação da rede serão positivamente influencia- } \\
\text { dos. }\end{array}$ & $\begin{array}{l}\text { Dyer e Singh (1998); Wang e Wei (2007); Gulati, Lavie e Singh (2009); Sarkar, } \\
\text { Aulakh e Madhok (2009); Swaminathan e Moorman (2009); Srai, Harrington e Ti- } \\
\text { wari (2016); Yeh (2016); Saunila et al. (2017); Weber et al. (2017); Wulf e Butel } \\
\text { (2017) }\end{array}$ \\
\hline $\begin{array}{l}\text { Novas parce- } \\
\text { rias }\end{array}$ & $\begin{array}{l}\text { Histórico de } \\
\text { parceria, Re- } \\
\text { putação da } \\
\quad \text { rede }\end{array}$ & $\begin{array}{l}\text { O estabelecimento de novas parcerias traz benefí- } \\
\text { cios à reputação da rede e elevam a possibilidade } \\
\text { de um histórico de parceria de sucesso. }\end{array}$ & $\begin{array}{l}\text { Wu e Cavusgil (2006); Gulati, Lavie e Singh (2009); Sarkar, Aulakh e Madhok } \\
\text { (2009); Swaminathan e Moorman (2009); Saunila et al. (2017) }\end{array}$ \\
\hline $\begin{array}{l}\text { Relaciona- } \\
\text { mento longo }\end{array}$ & $\begin{array}{l}\text { Investimento, } \\
\text { Satisfação, Si- } \\
\text { nergia }\end{array}$ & $\begin{array}{l}\text { O relacionamento com os mesmos parceiros ofe- } \\
\text { rece melhores benefícios, pois a integração é mais } \\
\text { estratégica, gerando retornos acima do esperado } \\
\text { devido à maior satisfação e sinergia. }\end{array}$ & $\begin{array}{l}\text { Kanter (1994); Dyer e Singh (1998); Madhok e Tallman (1998); Monge et al. (1998); } \\
\text { Holm, Eriksson e Johanson (1999); Johnson (1999); Gulati, Lavie e Singh (2009); } \\
\text { Swaminathan e Moorman (2009); Maurer (2010); Wagner, Eggert e Lindemann } \\
\text { (2010); Cao e Zhang (2011); Romero e Molina (2011); Bliemel, Mccarth e Maine } \\
\text { (2016); Bouncken e Fredrich (2016); Charterina, Basterretxea e Landeta (2016); } \\
\text { Hughes-Morgan e Yao (2016); Jraisat (2016); Sadovnikova e Pujari (2017); Saunila } \\
\text { et al. (2017) }\end{array}$ \\
\hline
\end{tabular}


Figura 4 - Lacunas da literatura

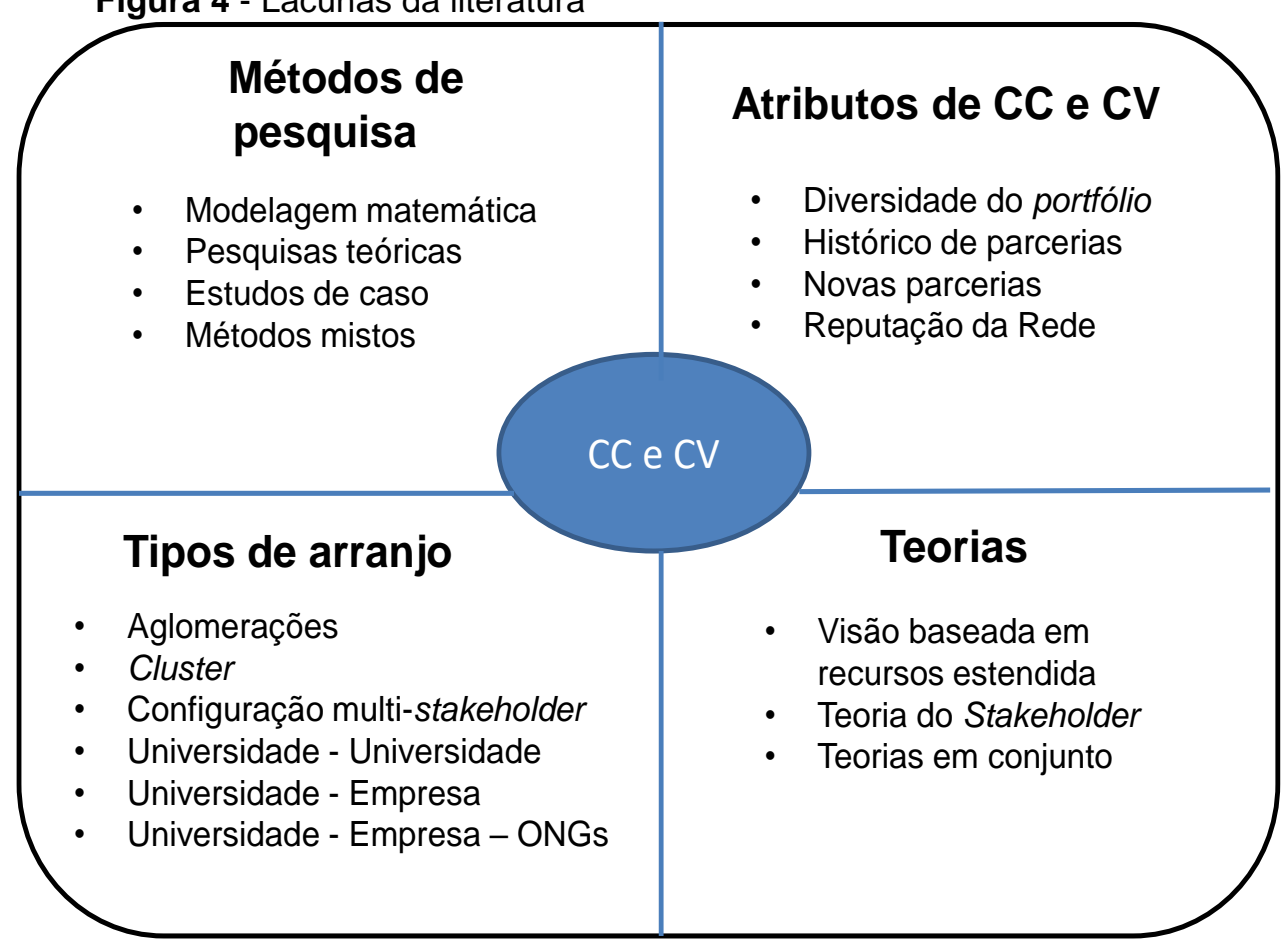

c) Teorias: Investigar a CC e a CV a partir de outras teorias. Por exemplo, a visão baseada em recursos estendida comumente é aplicada para estudar relacionamentos no contexto de cadeias de suprimentos. Sugere-se a aplicação dessa teoria para estudar a relacionamentos entre outras aglomerações (como clusters industriais). Além disso, estudar a CV e o impacto nas rendas relacionais a partir da visão relacional também merece uma maior investigação.

d) Atributos de CC e CV: Dentre os atributos analisados, quatro deles (Fig. 4) ainda são pouco explorados. Logo, futuras pesquisas podem focar nesses atributos de modo mais aprofundado. Além disso, a adoção de métodos mistos poderia auxiliar a compreender melhor a complexidade da temática. Por exemplo, aplicar uma pesquisa com especialistas para verificar quais atributos de CC influenciam na CV e, em seguida, desenvolver um modelo quantitativo por meio de modelagem matemática (metodologias multicritério) que possam auxiliar na tomada de decisão em relação às parcerias interorganizacionais.

\section{CONCLUSÃO}

Nos dias atuais, enfrentar com êxito questões globais, sem um entendimento e 
sem colaboração entre os diferentes atores de cada setor da sociedade, tem se tornado um problema. Dessa forma, a necessidade de colaborar a partir de diferentes relacionamentos interorganizacionais passa a ser um desafio gerencial devido a sua dificuldade e complexidade. Para obterem êxito, a CC das empresas passou a ser importante, pois a partir dela organizações podem CV conjunto.

Diante desse contexto, e a partir da RSL apresentada, verificou-se que pesquisas que exploram o relacionamento entre CC e CV no contexto de relacionamentos interorganizacionais ainda são escassas, sendo esta uma das contribuições desse artigo, ou seja, o aprofundamento da teoria de uma temática recente.

Este trabalho teve como objetivo identificar os atributos que influenciam no desenvolvimento de CC para a CV. Assim, outra contribuição diz respeito à identificação dos fatores de CC que influenciam nos atributos de CV (Quadro 4) que permite que gestores analisem de forma sistemática os atributos de CC que influenciam na CV, auxiliando-os a perceberem exatamente em qual aspecto/atributo eles precisam meIhorar para ampliar o seu potencial de CV conjunto, contribuindo para uma tomada de decisão acertada.

Por fim, a identificação das tendências de pesquisa, assim como gaps sinalizaram algumas oportunidades que ainda precisam ser explorados em termos de métodos, teorias, tipos de arranjos e atributos de CC e CV que precisam um maior aprofundamento. Essas oportunidades representam outra contribuição dessa pesquisa.

O artigo apresenta algumas limitações, sendo uma delas o método de RSL adotado. Dessa forma, novas pesquisas podem incluir outras bases de dados, outras palavras-chave ou atualizar a revisão e os atributos de CC e CV apresentados. Apesar da identificação dos atributos de CC que influenciam no desenvolvimento de CV no contexto de relacionamentos interorganizacionais, esta pesquisa não analisou a influência dessa relação, tampouco a quantificou. Dessa forma, novas pesquisas podem analisar essa influência, por meio de metodologias multicritério ou de métodos qualitativos, como o Delphi e o Estudo de Caso. Por fim, a análise do impacto desses atributos sobre o desempenho das parcerias por meio de Surveys podem surgir como uma possibilidade de novas pesquisas.

\section{REFERÊNCIAS}

ALVAREZ, S. A.; BARNEY, J. B. How entrepreneurial firms can benefit from alliances with 
large partners. Academy of Management Executive, v. 15, n. 1, p. 139-148, 1 fev. 2001. https://doi.org/10.5465/ame.2001.4251563
ARMITAGE, A.; KEEBLE-ALLEN, D. Undertaking a Structured Literature Review or
Structuring a Literature Review: .. of the 7th European Conference on ....
[Anais...]2008Disponível em:
http://books.google.co.uk/books?hl=en\&lr=\&id=3WJ6dTIr8iEC\&oi=fnd\&pg=PA35\&dq=meta- ethnography\&ots=rP0wf-196-\&sig=NqsuitzEykhoB09fJ13CrRJuJSA

BARROSO-MÉNDEZ, M. J. et al. Cross-sector social partnership success: A process perspective on the role of relational factors. European Management Journal, v. 34, n. 6, p. 674-685, dez. 2016. https://doi.org/10.1016/j.emj.2016.07.004

BLIEMEL, M. J.; MCCARTHY, I. P.; MAINE, E. M. A. Levels of Multiplexity in Entrepreneur's Networks: Implications for Dynamism and Value Creation. Entrepreneurship Research Journal, v. 6, n. 3, 1 jan. 2016. https://doi.org/10.1515/erj-2015-0001

BOUNCKEN, R. B.; FREDRICH, V. Business model innovation in alliances: Successful configurations. Journal of Business Research, v. 69, n. 9, p. 3584-3590, set. 2016. https://doi.org/10.1016/j.jbusres.2016.01.004

BRINKERHOFF, J. M. Government-nonprofit partnership: a defining framework. Public Administration and Development, v. 22, n. 1, p. 19-30, fev. 2002. https://doi.org/10.1002/pad.203

CAO, M.; ZHANG, Q. Supply chain collaboration: Impact on collaborative advantage and firm performance. Journal of Operations Management, v. 29, n. 3, p. 163-180, mar. 2011. https://doi.org/10.1016/j.jom.2010.12.008

CHEUNG, M.; MYERS, M. B.; MENTZER, J. T. Does relationship learning lead to relationship value? A cross-national supply chain investigation. Journal of Operations Management, v. 28, n. 6, p. 472-487, nov. 2010. https://doi.org/10.1016/j.jom.2010.01.003

CZAKON, W. Relational capability of organizations: Theoretical advances. Journal of Economics \& Management, v. 5, p. 48-65, 2009.

DELLYANAA, D.; SIMATUPANGB, T. M.; DHEWANTOC, W. Business model innovation in different strategic networks. International Journal of Business, v. 21, n. 3, p. 191-215, 2016.

DYER, J. H.; SINGH, H. The Relational view: Cooperative Strategy and Sources of Interorganizational Competitive Advantage. The Academy of Management Review, v. 23, n. 4, p. 660-679, 1998a. https://doi.org/10.5465/amr.1998.1255632

DYER, J. H.; SINGH, H. The Relational view: Cooperative Strategy and Sources of Interorganizational Competitive Advantage. The Academy of Management Review, v. 23, n. 4, p. 660-679, 1998b. https://doi.org/10.5465/amr.1998.1255632

GULATI, R.; LAVIE, D.; SINGH, H. The nature of partnering experience and the gains from alliances. Strategic Management Journal, v. 30, n. 11, p. 1213-1233, nov. 2009. https://doi.org/10.1002/smj.786

HANSEN, M. T.; NOHRIA, N. How to build collaborative advantage. Mit Sloan Management Review, v. 46, n. 1, p. 22- +, 2004. 
HARRISON, J. S.; BOSSE, D. A.; PHILLIPS, R. A. Managing for stakeholders, stakeholder utility functions, and competitive advantage. Strategic Management Journal, v. 31, n. 1, p. 58-74, jan. 2010. https://doi.org/10.1002/smj.801

HELFAT, C. E. et al. Dynamic Capabilities: understanding strategic changes in organizations. [s.I.] Blackwell Publishing, 2007.

HSIAO, Y.-C. et al. Resource alignment, organizational distance, and knowledge transfer performance: the contingency role of alliance form. The Journal of Technology Transfer, v. 42, n. 3, p. 635-653, 16 jun. 2017. https://doi.org/10.1007/s10961-016-9505-4

HUGHES-MORGAN, M.; YAO, B. E. Rent Appropriation in Strategic Alliances: A Study of Technical Alliances in Pharmaceutical Industry. Long Range Planning, v. 49, n. 2, p. 186195, abr. 2016. https://doi.org/10.1016/j.Irp.2015.12.016

JAP, S. D. Efforts : Processes in Collaboration Buyer-Supplier Relationships. Journal of Marketing Research, v. 36, n. 4, p. 461-475, 1999.

https://doi.org/10.1177/002224379903600405

JOHNSON, J. L. Strategic integration in industrial distribution channels: managing the interfirm relationship as a strategic asset. Journal of the Academy of Marketing Science, v. 27, n. 1, p. 4-18, 1 jan. 1999. https://doi.org/10.1177/0092070399271001

JONES, G. J. et al. Collaborative Advantages: The Role of Interorganizational Partnerships for Youth Sport Nonprofit Organizations. Journal of Sport Management, v. 31, n. 2, p. 148160, mar. 2017. https://doi.org/10.1123/ism.2016-0118

JRAISAT, LUAI. A network perspective and value added tasks: the case of agri-food value chain. Asia Pacific Journal of Marketing and Logistics, v. 28, n. 2, p. 350-365, 2016. https://doi.org/10.1108/APJML-08-2015-0124

KAUFFMAN, R. J.; LI, T.; VAN HECK, E. Business Network-Based Value Creation in Electronic Commerce. International Journal Of Electronic Commerce, v. 15, n. 1, p. 113143, 2010. https://doi.org/10.2753/JEC1086-4415150105

KLEIN, R.; RAI. ARUN. Interfirm Strategic Information Flows in Logistics Supply Chain Relationships. MIS Quarterly, v. 33, n. 4, p. 735, 2009. https://doi.org/10.2307/20650325

LACERDA, R.; ENSSLIN, L.; ENSSLIN, S. R. Uma análise bibliométrica da literatura sobre estratégia e avaliação de desempenho. Gestão \& Produção, v. 19, n. 1, p. 59-78, 2012. https://doi.org/10.1590/S0104-530X2012000100005

LADO, A. A; BOYD, N. G.; HANLON, S. C. and the Search Model for Economic Rents : a Syncretic. Management, v. 22, n. 1, p. 110-141, 1997. https://doi.org/10.5465/amr.1997.9707180261

LI, M.; ZHENG, X.; ZHUANG, G. Information technology-enabled interactions , mutual monitoring , and supplier-buyer cooperation: A network perspective. Journal of Business Research, 2017. https://doi.org/10.1016/j.jbusres.2016.12.022

LOEBBECKE, C.; FENEMA, P. C. VAN; POWELL, P. Journal of Strategic Information Systems Managing inter-organizational knowledge sharing. Journal Of Strategic Information Systems, 2016. https://doi.org/10.1016/j.jsis.2015.12.002 
MANI, S. et al. Stock Market and Network Influence on Alliance Formation : Evidence from the Biopharmaceutical Industry STOCK MARKET AND NETWORK INFLUENCE ON

ALLIANCE FORMATION : Journal of Marketing Theory and Practice, v. 25, n. 1, p. 69-85, 2017. https://doi.org/10.1080/10696679.2016.1236664

MATINHEIKKI, J. et al. ScienceDirect Managing inter-organizational networks for value creation in the front-end of projects. JPMA, v. 34, n. 7, p. 1226-1241, 2016.

https://doi.org/10.1016/j.ijproman.2016.06.003

MATUSIK, S. F.; HEELEY, M. B. Absorptive Capacity in the Software Industry: Identifying Dimensions That Affect Knowledge and Knowledge Creation Activities. Journal of Management, v. 31, n. 4, p. 549-572, 30 ago. 2005.

https://doi.org/10.1177/0149206304272293

MOLINA-MORALES, F. X.; MARTÍNEZ-FERNÁNDEZ, M. T. Too much love in the neighborhood can hurt: how an excess of intensity and trust in relationships may produce negative effects on firms. Strategic Management Journal, v. 30, n. 9, p. 1013-1023, set. 2009. https://doi.org/10.1002/smj. 766

MÖLLER, K. E. K.; TÖRRÖNEN, P. Business suppliers' value creation potential. Industrial Marketing Management, v. 32, n. 2, p. 109-118, fev. 2003. https://doi.org/10.1016/S0019$\underline{8501(02) 00225-0}$

MONGE, P. R. et al. Production of Collective Action in Alliance-Based Interorganizational Communication and Information Systems. Organization Science, v. 9, n. 3, p. 411-433, jun. 1998. https://doi.org/10.1287/orsc.9.3.411

PANDA, D. K. Public private partnerships and value creation: the role of relationship dynamics. International Journal of Organizational Analysis, v. 24, n. 1, p. 162-183, 14 mar. 2016. https://doi.org/10.1108/IJOA-12-2014-0830

PANGARKAR, N.; YUAN, L.; HUSSAIN, S. Too much of a good thing ? Alliance portfolio size and alliance expansion. European Management Journal, 2016.

https://doi.org/10.1016/j.emj.2016.09.006

PARUNG, J.; BITITCI, U. S. A conceptual metric for managing collaborative networks. Journal of Modelling in Management, v. 1, n. 2, p. 116-136, maio 2006.

https://doi.org/10.1108/17465660610703468

PARUNG, J.; BITITCI, U. S. A metric for collaborative networks. Business Process Management Journal, v. 14, n. 5, p. 654-674, 12 set. 2008.

https://doi.org/10.1108/14637150810903048

PERUCCI, C. C.; CAMPOS, F. C. Técnicas de Qualidade Aplicadas em Software: Um Estudo Bibliométrico. Revista de Ciência \& Tecnologia, v. 19, n. 38, p. 5-15, 30 dez. 2016. https://doi.org/10.15600/2238-1252/rct.v19n38p5-15

RÜHLI, E. et al. Innovation in Multistakeholder Settings: The Case of a Wicked Issue in Health Care. Journal of Business Ethics, v. 143, n. 2, p. 289-305, 11 jun. 2017. https://doi.org/10.1007/s10551-015-2589-1

SADOVNIKOVA, A.; PUJARI, A. The effect of green partnerships on firm value. Journal of the Academy of Marketing Science, 2016. https://doi.org/10.1007/s11747-016-0490-9 
SARAF, N.; LANGDON, C. S.; GOSAIN, S. IS Application Capabilities and Relational Value in Interfirm Partnerships. Information Systems Research, v. 18, n. 3, p. 320-339, set. 2007. https://doi.org/10.1287/isre.1070.0133

SARKAR, M. et al. The Influence of Complementarity, Compatibility, and Relationship Capital on Alliance Performance. Journal of the Academy of Marketing Science, v. 29, n. 4, p. 358-373, 1 out. 2001. https://doi.org/10.1177/03079450094216

SARKAR, M.; AULAKH, P. S.; MADHOK, A. Process Capabilities and Value Generation in Alliance Portfolios. Organization Science, v. 20, n. 3, p. 583-600, jun. 2009. https://doi.org/10.1287/orsc.1080.0390

SAUNILA, M. et al. Gaining insights into the measurement of value in industrial service network. International Journal of Quality \& Reliability Management, v. 34, n. 4, p. 478493, 3 abr. 2017. https://doi.org/10.1108/IJQRM-03-2015-0046

SIMATUPANG, T. M.; SRIDHARAN, R. An integrative framework for supply chain collaboration. The International Journal of Logistics Management, v. 16, n. 2, p. 257274, 2005. https://doi.org/10.1108/09574090510634548

STADTLER, H. A framework for collaborative planning and state-of-the-art. OR Spectrum, v. 31, n. 1, p. 5-30, 24 jan. 2009. https://doi.org/10.1007/s00291-007-0104-5

SUBRAMANI, M. How Do Suppliers Benefit from Information Technology Use in Supply Chain Relationships? v. 28, n. 1, p. 45-73, 2004. https://doi.org/10.2307/25148624

SWAMINATHAN, V.; MOORMAN, C. Marketing Alliances, Firm Networks, and Firm Value Creation. Journal of Marketing, v. 73, n. 5, p. 52-69, set. 2009.

https://doi.org/10.1509/jmkg.73.5.52

TANRIVERDI. Performance Effects of Information Technology Synergies in Multibusiness Firms. MIS Quarterly, v. 30, n. 1, p. 57, 2006. https://doi.org/10.2307/25148717

TEECE, D. J.; PISANO, G.; SHUEN, A. Dynamic capabilities and strategic management. The Nature and Dynamics of Organizational Capabilities, v. 18, n. 7, p. 334-361, 1997. https://doi.org/10.1002/(SICI)1097-0266(199708)18:7<509::AID-SMJ882>3.0.CO;2-Z

TELLER, C.; ALEXANDER, A.; FLOH, A. The impact of competition and cooperation on the performance of a retail agglomeration and its stores. Industrial Marketing Management, 2015. https://doi.org/10.1016/j.indmarman.2015.07.010

TORKKELI, L. et al. Network competence in Finnish SMEs: implications for growth. Baltic Journal of Management, v. 11, n. 2, p. 207-230, 4 abr. 2016. https://doi.org/10.1108/BJM02-2015-0032

VOLSCHENK, J.; UNGERER, M.; SMIT, E. Industrial Marketing Management Creation and appropriation of socio-environmental value in coopetition. Industrial Marketing Management, 2016. https://doi.org/10.1016/j.indmarman.2016.05.026

WAGNER, S. M.; EGGERT, A.; LINDEMANN, E. Creating and appropriating value in collaborative relationships. Journal of Business Research, v. 63, n. 8, p. 840-848, 2010. https://doi.org/10.1016/j.jbusres.2010.01.004 
WANG, E. T. G.; WEI, H.-L. Interorganizational Governance Value Creation: Coordinating for Information Visibility and Flexibility in Supply Chains*. Decision Sciences, v. 38, n. 4, p. 647-674, 7 dez. 2007. https://doi.org/10.1111/j.1540-5915.2007.00173.x

WHITE, M.; MARSH, E. Content analysis: A flexible methodology. Library Trends, v. 55, n. 1, p. 22-45, 2006. https://doi.org/10.1353/lib.2006.0053

WU, F.; CAVUSGIL, S. T. Organizational learning, commitment, and joint value creation in interfirm relationships. Journal of Business Research, v. 59, n. 1, p. 81-89, jan. 2006. https://doi.org/10.1016/j.jbusres.2005.03.005

ZHANG, S.; LI, N.; LI, J. Redefining relational rent. Technological Forecasting and Social Change, v. 117, p. 315-326, 2017. https://doi.org/10.1016/j.techfore.2016.10.072

ZHANG, X.; VAN DONK, D. P.; VAN DER VAART, T. The different impact of inter-organizational and intra-organizational ICT on supply chain performance. International Journal of Operations \& Production Management, v. 36, n. 7, p. 803-824, 2016.

https://doi.org/10.1108/lJOPM-11-2014-0516

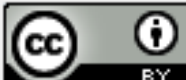

Artigo recebido em: 07/04/2018 e aceito para publicação em: 01/12/2019

DOI: http://dx.doi.org/10.14488/1676-1901.v19i4.3214 